\title{
Communications and networking over satellites: SatNEx experimental activities and testbeds
}

\author{
T. de Cola ${ }^{1, *, \dagger}$, L. Ronga ${ }^{2}$, T. Pecorella ${ }^{2}$, P. Barsocchi ${ }^{3}$, S. Chessa $^{3}$, E. Ferro $^{3}$, \\ A. Gotta ${ }^{3}$, G. Oligeri ${ }^{3}$, F. Potorti ${ }^{3}$, R. Secchi ${ }^{3}$, A. Sathiaseelan ${ }^{4}$, V. M. Castro ${ }^{5}$, \\ R.J. Peral ${ }^{5}$, C. Caini ${ }^{6}$ and R. Firrincieli ${ }^{6}$ \\ ${ }^{1}$ German Aerospace Center (DLR), Institute of Communications and Navigation, Wessling, Germany \\ ${ }^{2}$ CNIT, Universtiy of Florence, Italy \\ ${ }^{3}$ CNR-ISTI, Pisa, Italy \\ ${ }^{4}$ Department of Engineering, University of Aberdeen, Aberdeen, UK \\ ${ }^{5}$ University of Barcelona, Barcelona, Spain \\ ${ }^{6}$ University of Bologna, Bologna, Italy
}

\begin{abstract}
SUMMARY
One of the aims of the SatNEx (Satellite Network of Excellence, http://www.satnex.de) European project is to provide the scientific community that operates on satellite communications with measurement campaign results and evaluation tools, in order to support research activities that investigate problems in satellite communications at different levels of the OSI stack. In fact, the research activities developed in SatNEx address the most relevant aspects of satellite communications, such as channel modulation and coding schemes, access strategies, advanced networking, end-to-end quality of service, transport performance, delay tolerant networks, and new applications. Even if SatNEx is mainly devoted to satellite communications, integration of satellite and terrestrial wireless segments has also been taken into account. This paper presents the results obtained in the specific research activity devoted to trials and testbeds developed during the SatNEx project. The high number of authors of this paper is an indication of the strong collaborative work sustained, the numerous measurement campaigns, and the complexity in developing integrated testbeds. Copyright (C) 2008 John Wiley \& Sons, Ltd.
\end{abstract}

Received 6 January 2008; Revised 23 July 2008; Accepted 28 July 2008

KEY WORDS: satellite communications; testbeds; measurement tools; performance evaluation

\section{INTRODUCTION}

The increasingly raising demand for broadband multimedia services to be offered seamlessly and ubiquitously to both mobile and fixed users has highlighted over the last years the important role jointly played by satellite and wireless technologies. They are consolidated as

\footnotetext{
*Correspondence to: Tomosco de Cola, German Aerospace Center (DLR), Institute of Communications and Navigations, D-82234 Weßling, Germany.

†E-mail: tomaso.decola@dlr.de

Contract/grant sponsor: IST FP6; contract/grant number: SatNEx (507052) and SatNEx II (027393).
} 
excellence means to enable communications anywhere and anytime [1]. From this point of view, the integration of telecommunication infrastructures built upon satellite and terrestrial wireless technologies has been assuming a primary importance [2] and pushing different companies to promote and offer quadruple-play services (high-speed Internet access, television, telephone, over wireless technologies) to mobile terminals, thus extending the range of interested customers that originally was restricted to fixed terminals only. In this perspective, it is immediate to see that the role of satellite technology is prominent in bridging the different actors involved in this scenario, owing to its intrinsic broadband and broadcast capabilities, suitable to deliver data and multimedia services.

From this overview, it is straightforward to see that this scenario environment offers interesting perspectives not only to companies that sell multimedia and broadband services, but also to the scientific community, due to the research challenges that may rise in terms of both communication and networking design.

The research activities performed by the SatNEx community perfectly fit in this philosophy; more precisely, SatNEx's goal is not only to provide the theoretical background necessary to properly investigate all the aforementioned communication and networking issues, but also to validate the effectiveness of protocol candidates identified during the preliminary study phase [2]. To this end, in the SatNEx framework, research activities devoted to measurement campaigns and development of evaluation tools have been carried out: the overview of these activities is the main focus of this paper. In more detail, this paper shows the validation methodologies used in SatNEx, aimed at evaluating protocols and architectures involved in satellite data communications.

Actually, evaluation tasks can be carried out through several viable approaches that can be classified as simulation, emulation, and trials on the field, which differentiate one from another in terms of complexity and accuracy in reproducing real environments. Indeed, although within the SatNEx project many research activities have been (and currently are) carried out by means of simulations, this paper addresses the measurement campaigns done on real platforms and the development of integrated testbeds. However, in some cases, measurements proved useful to validate the accuracy of simulation models. The aforementioned approaches will be hereafter referred to as experiments.

Given the high complexity of the satellite communications, the experimentation framework can be suitably subdivided into two different parts: the reference scenario and the protocol architecture evaluation (Figure 1). The former characterizes the environment peculiarities, in order to point out the main factors that could affect the protocol performance. The latter directly addresses the protocol and architecture performance evaluation, and can actually be subdivided into two main levels. At the bottom, access, network, and transport issues are considered in a single framework, which may imply cross-layer investigation and optimization, as demanded for in the most advanced terrestrial wireless and satellite systems. At the top, the application is actually conceived to define services (e.g. audio, video conference) to be handled by the protocol entities of underlying layers. In this case, the 'quality' of video/audio services and the effectiveness of the signalling mechanisms, thereby applied to establish the multimedia conference sessions, have to be evaluated.

The activity that we present has been carried out by means of suitable investigation tools, specifically devised to allow the accurate analysis of several aspects in the field of satellite communications, as pointed out later on. In fact, the methodology adopted to conduct tests was two-fold and reflected the peculiarities and features offered by the different tools: 


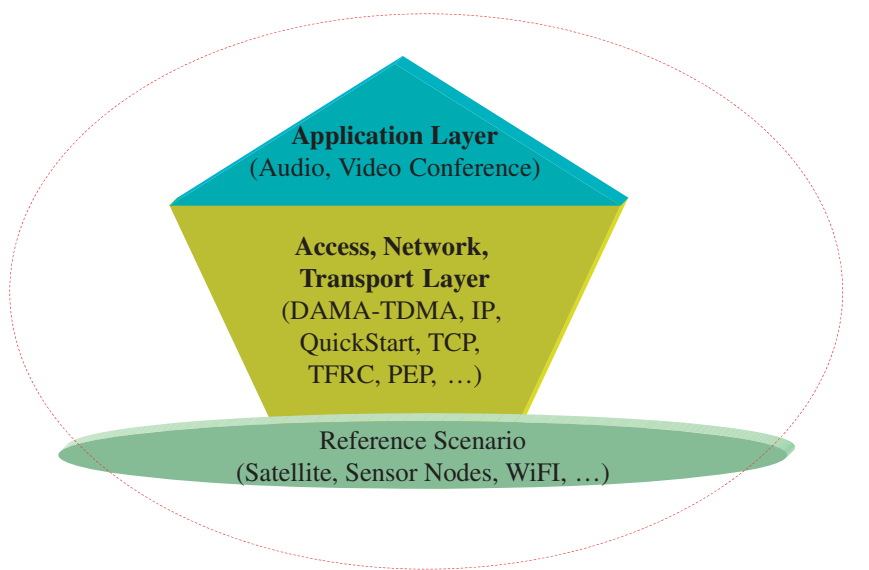

Figure 1. The global experimentation framework, enclosed in the circle.

measurement campaigns, and the use of emulation tools and their integration with real platforms. In the first case, we describe the experimental environment, the measurement campaign itself, and the most prominent results obtained. In the second case, we describe the integrated testbeds, the facilities they offer, and also a limited set of results, to highlight their powerful analysis features. In both cases, the interested reader is referred to the cited literature for an exhaustive analysis.

The paper is organized as follows. Section 2 introduces the reference scenario and how the presented experiments are positioned in it. Section 3 briefly describes the Skyplex satellite platform used in most of the experiments. Section 4 illustrates the measurement campaigns, whereas Section 5 is devoted to the description of the integrated testbeds. Finally, Section 6 draws the conclusions of this work and indicates possible extensions for the future.

\section{THE REFERENCE SCENARIO}

The focus of the experimentations envisioned in the SatNEx project is on heterogeneous networks, with a particular attention to the role of satellites, seen as fundamental means to guarantee widespread coverage and broadband multimedia communications. Satellite links have to ensure interconnectivity among networked islands, which can be built upon cabled and wireless technologies for their intrinsic capability of connecting people at moderate costs. This general view must also consider the extension of common Internet infrastructures toward space exploration applications. In this perspective, also interplanetary links are considered in this wide scenario: links connecting local Earth data gathering centers and remote stations, located on Mars or the Moon, are assumed. In particular, the case of remote interplanetary sensing applications, performed by sensor nodes and remotely controlled by Earth stations, is taken as a reference. The global scenario investigated is shown in Figure 2. 


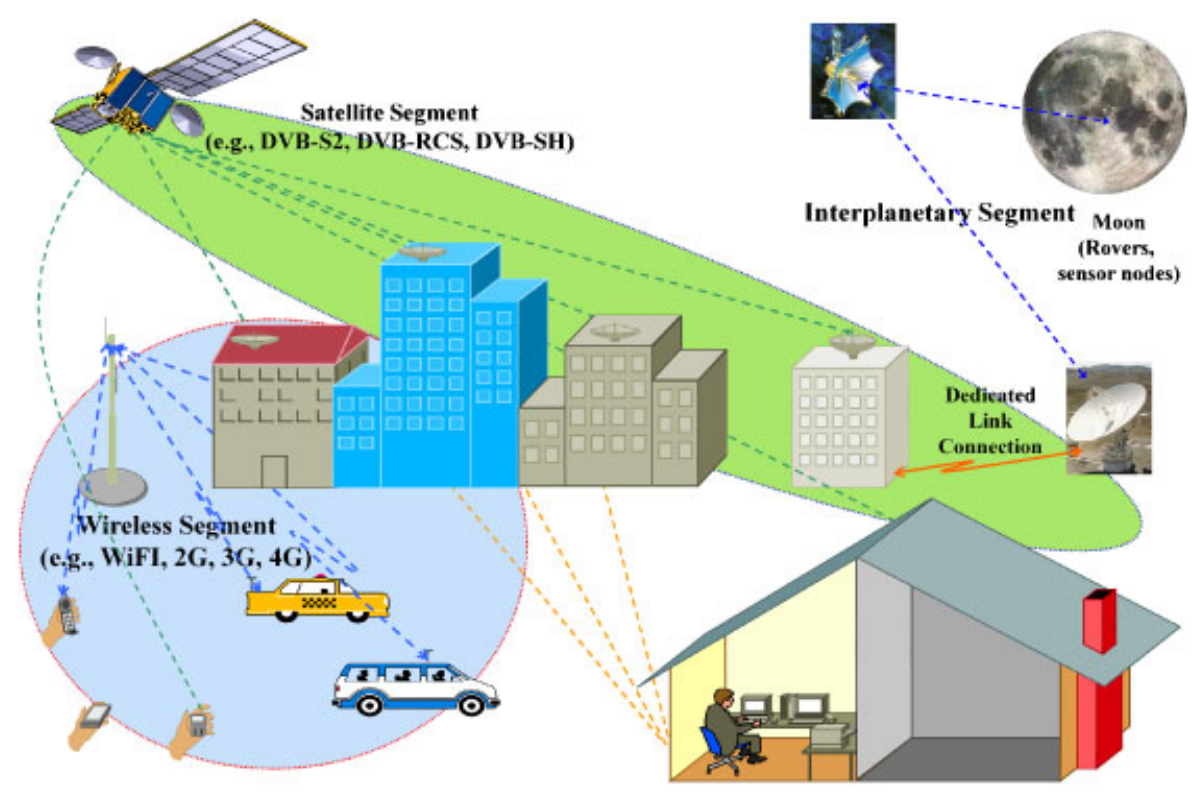

Figure 2. The reference scenario investigated.

Starting from the reference scenario sketched in Figure 2, it is straightforward to see that different investigations tools are required to analyze the various communication and networking challenges exhibited by such environment. In fact, the experiments that we present in this paper have been performed by means of a combination of trials on the field, emulation, and, sometime, simulation plus validation through trials on the field.

The experiments are as follow:

- VOTOS (various TCPs on satellite) analyzes the performance of some versions of TCP protocols when both data and real time traffic are sent together on the satellite link. VoIP over satellite exclusively addresses VoIP applications running on different platforms, by focusing on the signalling solutions adopted to establish the conference sessions, and, more important, on typical metrics employed while analyzing quality of service issues (e.g. delay, jitter, etc.).

- WICHMO (wireless channel modelling) derives a model for outdoor Wi-Fi propagation from both a significant amount of outdoor measurements on the Wi-Fi channel in an interconnected wireless-satellite environment.

- UCIT (UoB-CNIT integrated testbed) and EGGS (satellite networking in challenging environments) aimed to develop testbeds suitable to investigate communication performance in two different environments. The former was devised for studying some specific aspects of interplanetary links, and it is focused on the delay tolerant network (DTN) architecture; as dealing with deep space communications, for which performing real measurements is impossible, EGGS relies on an emulation of the links characteristics. UCIT deals with the evaluation of advanced transport protocol and architectures over heterogeneous networks, which includes satellite links. As addressing satellite communications, UCIT can either rely on emulated channels or real satellite platforms. 


\section{THE REAL SATELLITE PLATFORM}

In all the experiments that involve a real satellite platform, the Skyplex data platform was used; it is based on the Skyplex OBP (on-board processing) technology run by Eutelsat on the HotBird 6 satellite [3], and it implements DVB-RCS features, while not being fully DVB-RCS compliant. It carries four Ka band transponders equipped with a Skyplex unit; the units are composed of channels configurable in low rate (at $2.112 \mathrm{Mbit} / \mathrm{s})$ or high rate (at $6.226 \mathrm{Mbit} / \mathrm{s}$ ) mode. The channels can be configured in single channel per carrier or time division multiple access (TDMA) mode, capacity sharing only being available in the second case. In TDMA mode, the frame structure is composed by $N$ time slots, occupied by bursts; each burst contains $M$ MPEG cells, thus $N \times M$ cells per frame are hosted. In such a structure, $N$ also represents the number of users per frame and defines the Skyplex TDMA Mode. In order to avoid the heavy constraint that only up to $N$ users can share the channel (i.e. one burst per frame assigned to each terminal) the frame structure has been extended by grouping together $L$ frames in a multiframe. Assigning, as a minimum, one burst per multi-frame to each user, the number of users per channel becomes $N \times L, L$ being the low rate, with a minimum granularity in our system of $44 \mathrm{kbit} / \mathrm{s}(N=6, M=8, L=8)$ instead of $352 \mathrm{kbit} / \mathrm{s}(N=6, M=8, L=1)$ for a single frame structure. In fact, the burst configuration present on the satellite platform referenced in this paper corresponds to $N=6, M=8, L=8$. Finally, three multi-frames constitute a super frame defined for signalling purposes. In our configuration, the super frame length results to be $820 \mathrm{~ms}$ long, which is the period of retransmission and validity of the schedule for the assignment of the multi-frame time slots to the terminals (burst time plan-BTP).

Bandwidth assignment can be either dynamic (demand assignment multiple access, DAMA), or static (continuous rate assignment) or mixed. In the first case, each terminal periodically requires the bandwidth on the basis of its own instantaneous need, and the time slots are assigned in a best-effort mode. The DAMA assignment does not provide any priority among terminals or any quality of service. In case of static allocation, a fixed number of time slots is pre-configured and assigned to a particular terminal.

\section{THE TRIALS ON THE FIELD EXPERIMENTS: VOTOS, VOIP OVER SATELLITE, WICHMO}

\subsection{VOTOS: Description of the experiment}

When satellite links interconnect terrestrial wireless networks, TCP dynamics are dominated by link errors, whose negative effects are amplified by the large delay-bandwidth product. The aim of the VOTOS experiment is to analyze the performance of TCP congestion control over a satellite DAMA (demand allocation multiple access) system.

A first part of VOTOS [4] consists in evaluating how standard New Reno TCP fares when compared with several other TCP variants, such as SACK [5] and Westwood + [6], a sender-only modification of TCP. The steady-state behavior of the Linux versions of these TCP flavors were studied in [4] over the Skyplex testbed, in a scenario that combines a satellite channel introducing a long delay and a WLAN segment experiencing channel errors. All versions have been tested in pairs (one connection per type), over the same path, in order to verify the interprotocol fairness. 
A second part of VOTOS deals with the startup phase of TCP, as opposed to steady state. With the diffusion of DAMA satellite systems, congestion control algorithms (such as TCP, TFRC, etc.) are increasingly going to experiment poor performance at startup, because the slow start phase is slowed down by DAMA. In fact, in rate-based DAMA systems, the bandwidth is assigned on the basis of the stations' requests, which, in turn, depend on the current transmission rates. Requests from the stations undergo a $250 \mathrm{~ms}$ propagation delay on geostationary satellite networks, and the allocation needs an additional $250 \mathrm{~ms}$ to be broadcast to the stations. This means that assignments are always late with respect to incoming traffic by at least $500 \mathrm{~ms}$, to which significant management overheads are usually added. Since the throughput in the slow start phase typically increases at each round trip time (RTT), the allocated bandwidth is always less than the offered traffic, which accounts for the very long slow start phase we observed in DAMA systems [7].

A third part of VOTOS considers possible solutions for the slow startup when multimedia traffic is involved. With the advent of audiovisual streaming on the Internet, congestion control mechanisms, such as TCP friendly rate control (TFRC) [8], will be mandatory for multimedia traffic. Since these algorithms emulate the behavior of TCP during the start-up phase, performance degradation similar to that of TCP is observed on DAMA links for these protocols. This means that the startup slowdown on DAMA systems affects both short TCP connections, like web browsing, and streaming applications using TFRC ) in the first few seconds of streaming, the most critical for fast switching between channels.

Other than using the Skyplex platform, we studied the interaction of quick start (QS) with TFRC and DAMA by using the ns-2 simulator. Since no DAMA support is available in ns-2, we developed a module for simulating a DAMA controlled satellite network [9]. The testbed was used to validate the DAMA module for the ns-2 simulation.

4.1.1. Results derived from the measurement campaign. TCP in steady state: First results are relative to fairness measured in steady-state conditions. We can make some observations on the measurements, shown in Table I. First, Westwood+with SACK exhibited a more aggressive behavior versus New Reno with SACK (trial 4) with respect to goodput in quasi error-free conditions. In trial 3, Westwood ${ }^{+}$appeared to be more robust than New Reno with respect to frame error rate (FER), with a higher goodput and less timeouts. In trials 1 and 2, which exhibited the most severe fading conditions, New Reno with SACK achieved the highest normalized goodput. New Reno SACK, the most diffused TCP flavor, was thus able to provide good performance in its Linux version. Westwood+SACK appeared to provide slightly better performance.

TCP startup: These results are relative to the startup phase of a single TCP connection over a lossless satellite hop. Figure 3 shows the first $20 \mathrm{~s}$ of acknowledged sequence numbers for the Linux implementations of the TCP stack, reporting 9 connection traces. The same happens with FreeBSD's TCP, though Linux is quicker because it sets the initial window to three packets (according to RFC 3390) while FreeBSD operating system more conservatively uses an initial window of one packet only.

We can distinguish two phases: an approximately exponential increase in sequence number followed by a linear increase phase. Indeed, since during the Slow Start phase the congestion window is increased by twice the amount of acknowledged segments, the ACK reception rate increases exponentially until the TCP throughput reaches the available channel capacity; after that point, ACK rate remains constant. However, we noticed that the time to double the 
Table I. Mean and median of: RTT, congestion window, and normalized goodput; Wi-Fi frame error rate; number of time outs (T.O.).

\begin{tabular}{|c|c|c|c|c|c|c|}
\hline \multirow[b]{2}{*}{$\begin{array}{l}\text { Trial } \\
\text { number }\end{array}$} & \multirow[b]{2}{*}{$\begin{array}{c}\text { TCP } \\
\text { variant }\end{array}$} & \multirow[b]{2}{*}{$\begin{array}{l}\text { Wireless } \\
\text { FER }\end{array}$} & \multicolumn{3}{|c|}{ Mean, median } & \multirow[b]{2}{*}{$\begin{array}{c}\text { Number of } \\
\text { timeouts }\end{array}$} \\
\hline & & & RTT (s) & $\begin{array}{c}\text { CWND } \\
(\mathrm{MSU}=1500 \mathrm{~B})\end{array}$ & $\begin{array}{l}\text { Normalized } \\
\text { goodput }\end{array}$ & \\
\hline \multirow[t]{2}{*}{1} & New Reno & $2.6 \times 10^{-3}$ & $1.56,1.23$ & $59.6,50.2$ & $0.32,0.38$ & 16 \\
\hline & $\begin{array}{l}\text { New Reno } \\
\text { SACK }\end{array}$ & $3.3 \times 10^{-3}$ & $2.03,1.37$ & $119.0,63.0$ & $0.39,0.42$ & 11 \\
\hline \multirow[t]{2}{*}{2} & Westwood + & $4.6 \times 10^{-3}$ & $1.83,1.24$ & $101.0,54.0$ & $0.31,0.33$ & 21 \\
\hline & Westwood+SACK & $3.9 \times 10^{-3}$ & $1.72,1.09$ & $78.0,45.0$ & $0.32,0.32$ & 12 \\
\hline \multirow[t]{2}{*}{3} & New Reno & $1.4 \times 10^{-3}$ & $1.90,1.30$ & $58.9,55.0$ & $0.27,0.28$ & 21 \\
\hline & Westwood+ & $1.1 \times 10^{-3}$ & $1.65,1.34$ & $58.3,56.0$ & $0.30,0.28$ & 11 \\
\hline \multirow[t]{2}{*}{4} & $\begin{array}{l}\text { New Reno } \\
\text { SACK }\end{array}$ & $3.1 \times 10^{-4}$ & $3.99,3.99$ & 196,170 & $0.46,0.45$ & 1 \\
\hline & Westwood+SACK & $4.3 \times 10^{-4}$ & $4.05,4.10$ & 213,224 & $0.50,0.52$ & 2 \\
\hline
\end{tabular}

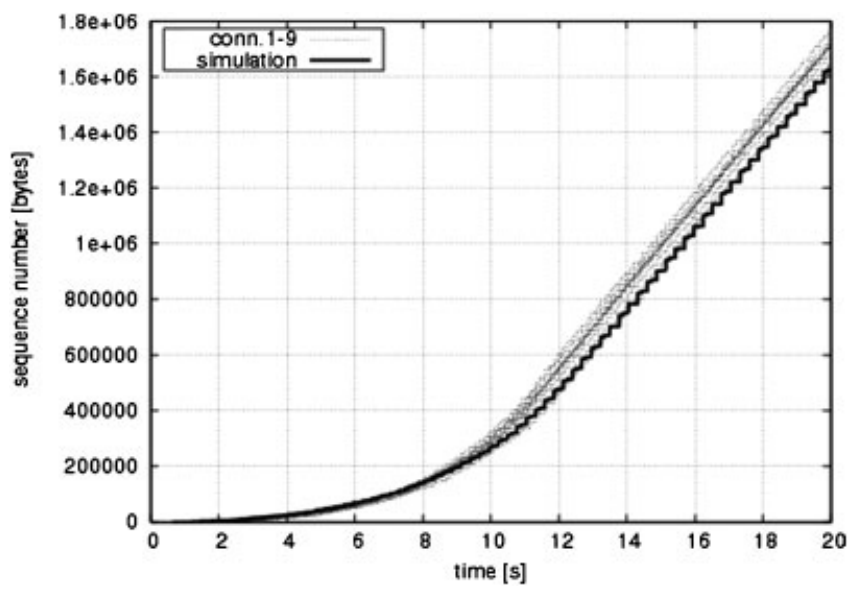

Figure 3. Startup behavior for Linux TCP.

congestion window is considerably longer than the connection RTT usually observed over terrestrial networks. This phenomenon should be attributed to the DAMA scheme adopted to share the satellite bandwidth.

To describe the interaction between the DAMA bandwidth assignment and the TCP startup dynamics, a simple approximate analytical model of the startup of a single TCP connection has been developed in [7]. This model points out that the Slow Start evolution in the presence of DAMA is equivalent to the one that would occur without DAMA, but with a longer RTT. In other words, the DAMA link, from the point of view of a TCP connection during the Slow Start phase, behaves like a fixed-bandwidth link with a longer equivalent propagation delay. In the case of the Skyplex platform, where the propagation delay is $310 \mathrm{~ms}$, measurements showed an equivalent propagation delay of $520 \mathrm{~ms}$, which is consistent with the model's results. 

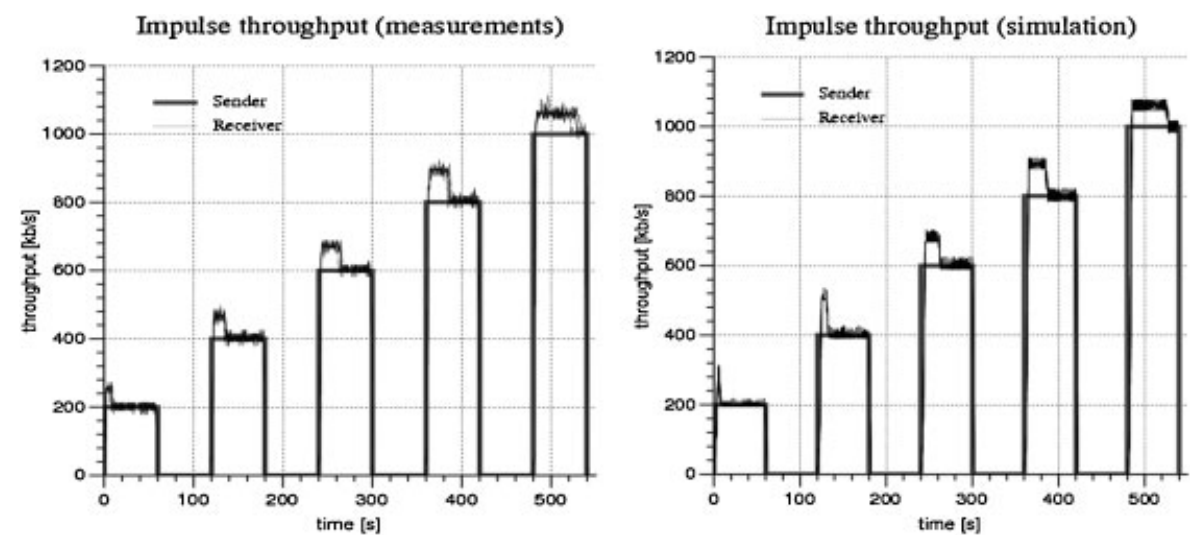

Figure 4. Measured and simulated throughput with offered impulse traffic.

This means that short TCP connections, which never reach steady state, such as most HTTP requests, are considerably slowed down on a DAMA allocation system than on a fixedassignment one.

In order to better understand the interactions between DAMA and TCP, we developed a DAMA simulation module for ns-2. The Skyplex platform was used to validate the model by comparing simulated traces with real traffic ones. In particular, we set up a unicast connection between two hosts, running Linux 2.6, one located at CNR-ISTI in Pisa and the other one at the CNIT Research Unit in Pisa. In order to carry out measurements of one-way delay, we synchronized the two hosts by using the network time protocol, which provided an accuracy of $10 \mathrm{~ms}$ for the delay estimation.

A total of eight simulation runs, each with a different offset of the bandwidth request with respect to the beginning of the TDMA frame (thus covering all possible cases), were performed. Figure 4 shows the response of the Skyplex network to impulses of UDP traffic in terms of throughput. UDP protocol has been used to generate traffic at different desired rates in order to validate the ns-2 simulation module. Graphs are depicted for both ns-2 simulation results and real trials on Skyplex. As detailed in [9], the response to an offered traffic impulse is a throughput transient with length depending on the DAMA parameters and amplitude proportional to the pulse height. Comparison of measured data with simulated ones yields excellent agreement.

Both real measurements and simulation confirmed that the interaction of DAMA with the TCP startup phase causes significant performance degradation. One possible approach to reduce it [10] is using the QS mechanism [11]. QS is an Internet engineering task force experimental protocol designed to provide lightweight signalling to the level of congestion (specifically available capacity) between routers and a pair of communicating end hosts.

\subsection{VoIP over satellite: description of the experiment}

The VoIP experiment is oriented to the exploration of those issues relevant to the usage of IPoriented voice services over geostationary satellites. The measured indexes show a relationship 
between quality metrics and the resulting user perception, when different configurations, voice codecs, and services are adopted.

The following VoIP services have been evaluated over the satellite link:

- single VoIP call between remote users;

- multiple VoIP calls among remote users;

- VoIP conferencing with mixed population (local and remote).

The experiments have been conducted with various satellite network loads, in order to inspect the impact of the combination of large delays and various packet loss rates.

The basic testbed adopted in the experiments is shown in Figure 5.

A local software VoIP PABX was installed in each satellite site. This choice was due to the requirement of coexistence between site local and remote voice services. The experiments collected statistical data of the user perception on the call quality and other objective performance indexes, such as packet arrival times and jitter, packet loss, bandwidth usage, and signalling (connection and tear-down) events.

The software used can be divided into three main categories: (1) VoIP PABX; (2) Soft Phones; (3) Traffic analysis.

Among the VoIP PABX software we tested three typical solutions, as follows:

(1) Linux Debian with Asterisk [12, 13] installed, the most popular open source telephone system in the world;

(2) Trixbox [14], an IP-PBX software solution designed for small and medium-sized businesses;

(3) AsteriskNOW beta5 [15], which brings the power and flexibility of Asterisk to a far broader group of customers.

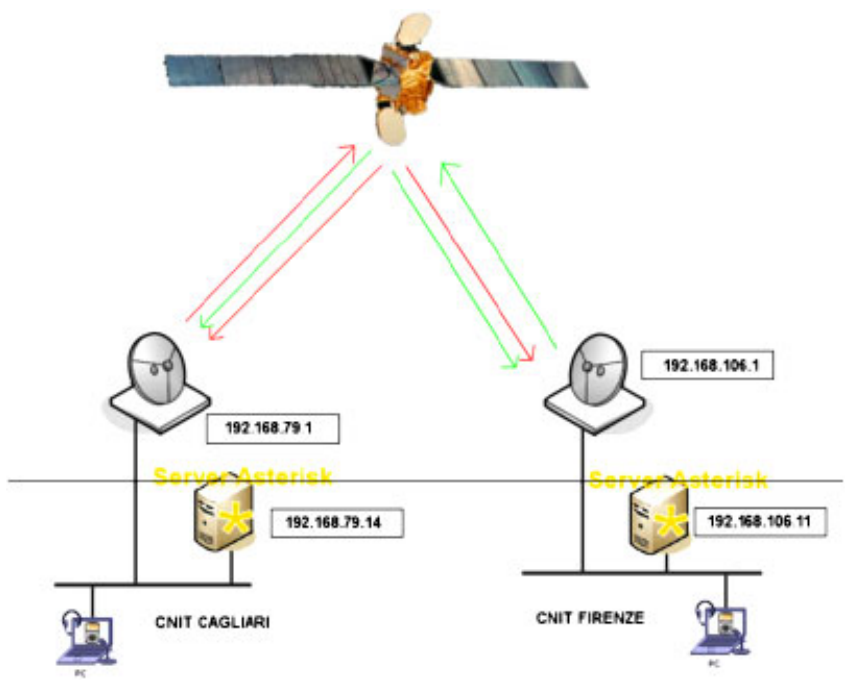

Figure 5. VoIP: basic experimental testbed. 
The three server systems are very similar, as they are built around the Asterisk VoIP PABX server system; however, some important differences must be noted. The first solution leads to the highest degree of configuration on what is running on the server, but also to the highest probability of misconfiguration and software conflicts. Trixbox is a well-known integrated solution for Asterisk, with auto-installation features. However, its components are not fully integrated; hence, for instance, accounting management and billing operations can be cumbersome at first. The user interface, moreover, is not uniform among Trixbox parts.

AsteriskNOW beta5 is the latest product from Digium (makers of Asterisk) and, though still in beta stage, it seems to be the most powerful, easy and clean web-based interface for Asterisk. AsteriskNow comes with self-installing features and a customized Linux OS (rPath Linux, [16]). Due to the built-in feature set available in AsteriskNOW (e.g. conference calls, web-based management, and hardware devices integration), all the software tests have been made on this platform.

The SoftPhones used are of two kinds, according to the protocol adopted. A number of SoftPhones have been evaluated, but the results have not shown any real audio quality difference among them. The main differences are the user interfaces, the codec availability, and the protocols supported $\left(\mathrm{SIP}^{\ddagger} / \mathrm{IAX}^{\S}\right)$. For the tests two SoftPhones have been selected, namely, Xlite [17] and Idefisk [18], shown in Figure 6. Finally the system's performance (delay, jitter, and bandwidth) was analyzed by using the network protocol analyzer WireShark (ex-Ethereal) [19]. It should be noted that WireShark can trap SIP and real-time transport protocol (RTP) packets, and can provide statistics based on SIP and RTP fields, but cannot perform similar operations with IAX, due to its binary in-channel signalling structure.

4.2.1. Results. A set of experiments has been carried out with both G.711u and GSM codecs. Results show that the G.711u average bandwidth occupation is $69.02 \mathrm{kbit} / \mathrm{s}$ with a standard deviation of $18.34 \mathrm{kbit} / \mathrm{s}$. The distribution of the bandwidth occupancy is shown in Figure 7 . This is particularly interesting for DBA dynamic bandwidth assignment (DBA) systems, since the non-constant bandwidth can trigger different DBA requests. On the contrary the GSM codec bandwidth usage is almost constant due to the missing silence suppression feature.

As far as propagation delays and jitter are concerned, exponential estimation techniques have been employed in order to overcome the lack of a tight synchronization between sender and receiver. Essentially, the estimation procedure works on time instants in which RTP packets are received and are generated, by taking advantage of the timestamp field present within RTP packet headers.

In more detail, let $R_{i}$ and $S_{i}$ denote the receiving time of the $i$ th packet and the timestamp value contained in the header of the related RTP packet, respectively.

\footnotetext{
Session Initiation Protocol.

${ }^{\S}$ Inter Asterisk Xchange. It is a protocol used by Asterisk, a server PBX open source of Digium. It is used to enable VoIP connections among Asterisk servers and among servers and clients that utilize the same protocol.

"Wireshark is the world's foremost network protocol analyzer, and is the de facto (and often de jure) standard across many industries and educational institutions.

"Real-time transport protocol (or RTP) defines a standardized packet format for delivering audio and video over the Internet.
} 

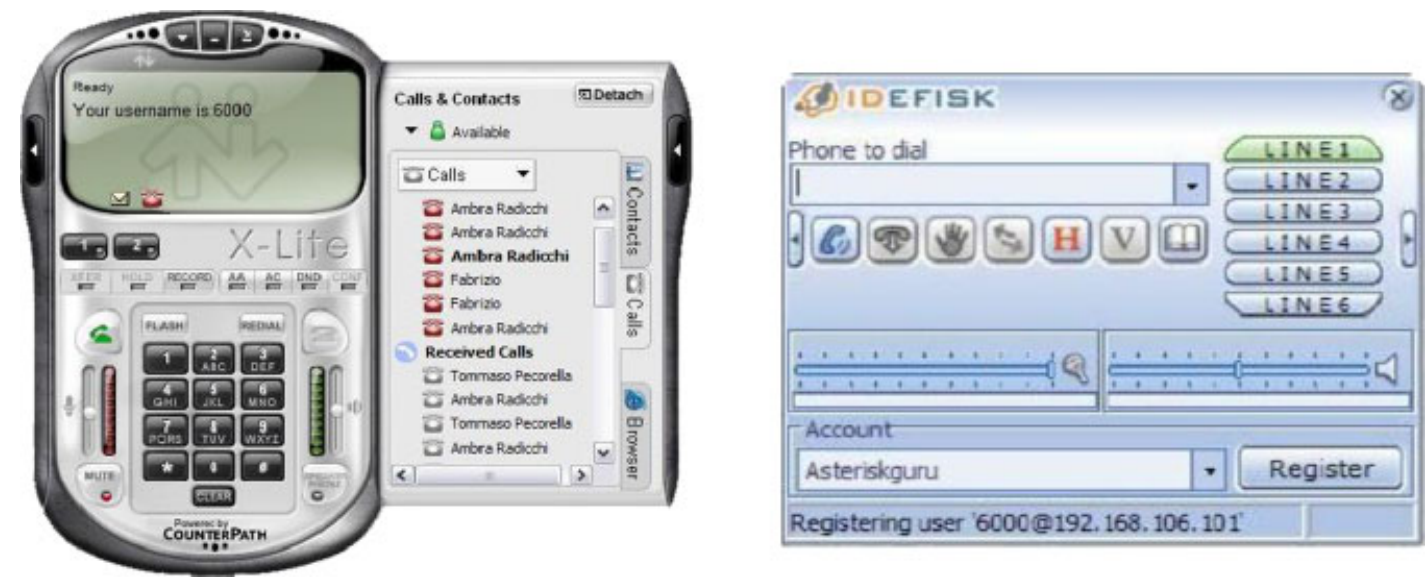

Figure 6. VoIP: SoftPhones used in the tests: Xlite on the left, Idefisk on the right.

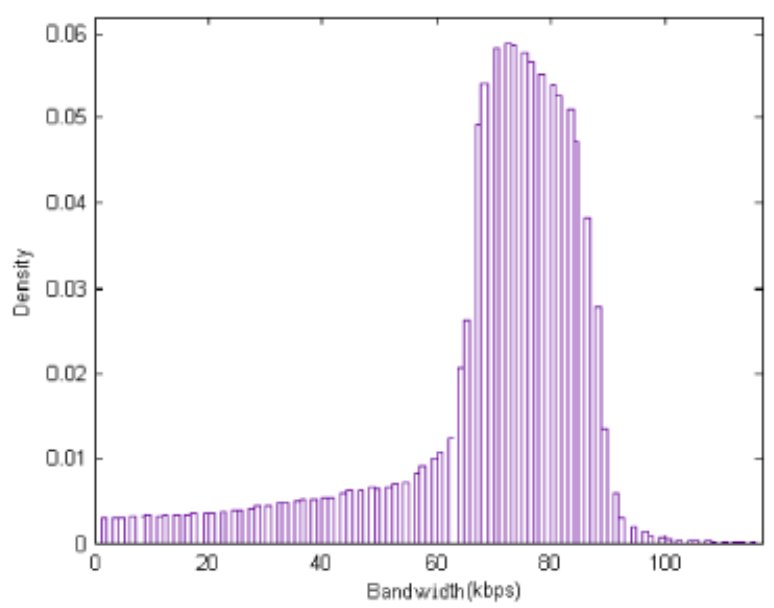

Figure 7. VoIP: bandwidth occupancy, G.711u codec.

Let's define $D(i, j)$ as in (1):

$$
\begin{aligned}
D(i, j) & =\left(R_{j}-R_{i}\right)-\left(S_{j}-S_{i}\right) \\
& =\left(R_{j}-S_{j}\right)-\left(R_{i}-S_{i}\right)
\end{aligned}
$$

It is immediate to see from $(1)$ that $D(i, j)$ compares the length of time intervals in which the $i$ th and $j$ th RTP packets are generated and received, respectively. In fact, this measure is a rough indication of the instantaneous jitter exhibited by the system in this observation interval.

Hence, from (1) it is finally possible to give an estimation of the jitter. To this end, let $J_{i-1}$ denote the jitter estimation performed at the arrival time of the $(i-1)$ th RTP packet. Then, the jitter estimation for the following RTP packet has to be evaluated by taking into account also 

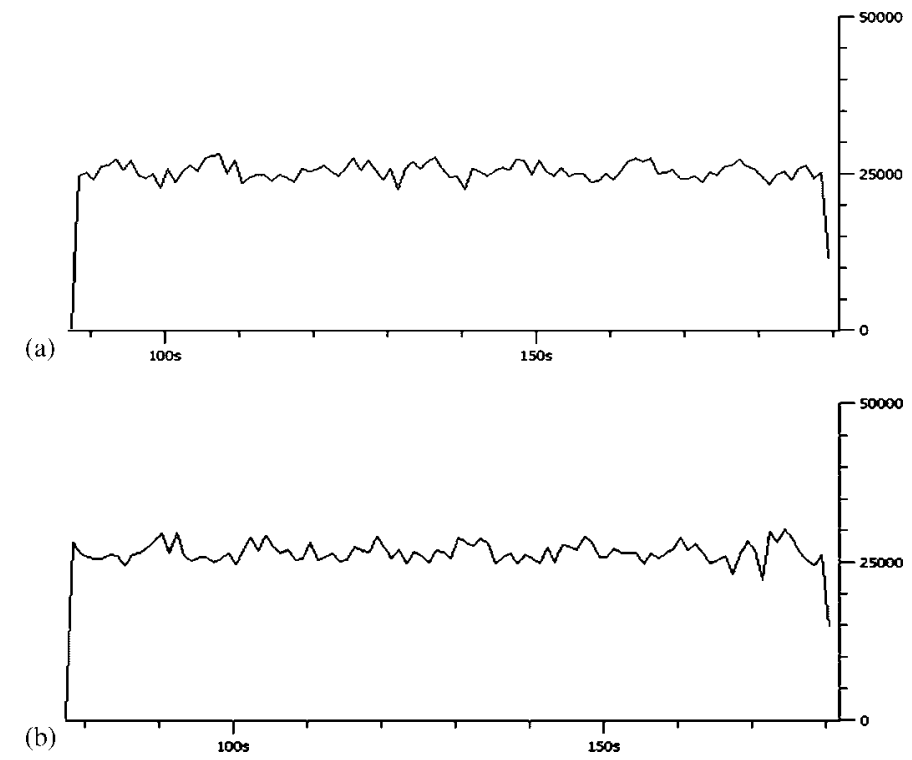

Figure 8. VoIP: SIP (8.a) and IAX (8.b) bandwidth occupancy ( $Y$ axis, expressed in bit/s) versus time ( $X$ axis). GSM codec.

Table II. MOS rating with different codecs and at different bit rates of the UDP interfering traffic, compared with standard MOS.

\begin{tabular}{lcccccc}
\hline Tested codec & Standard MOS & $1.4 \mathrm{Mbit} / \mathrm{s}$ & $1.2 \mathrm{Mbit} / \mathrm{s}$ & $1.1 \mathrm{Mbit} / \mathrm{s}$ & $0.75 \mathrm{Mbit} / \mathrm{s}$ & $0.35 \mathrm{Mbit} / \mathrm{s}$ \\
\hline GSM & $3.7 \div 3.9$ & $\mathrm{X}$ & LAGGED & 2 & $2 \div 3$ & 3 \\
G.711a & 4.4 & $\mathrm{X}$ & LAGGED & $2 \div 3$ & 3 & 4 \\
ILBC & $3.7 \div 4.1$ & $\mathrm{X}$ & LAGGED & $2 \div 3$ & 3 & $3 \div 4$ \\
\hline
\end{tabular}

the instantaneous jitter measured in this time interval, by applying (2)

$$
J_{i}=J_{i-1}+\left(|D(i-1, i)|-J_{i-1}\right) / 16
$$

Basically, (2) performs a sort of low-pass filtering operations on instantaneous jitter samples in order to cope with possible synchronization misalignments between source and destinations. It was shown that the obtained estimation is a good approximation of the real jitter.

The measured jitter introduced by the network has an average of $33.1 \mathrm{~ms}$ and a standard deviation of $4.59 \mathrm{~ms}$; this is perfectly compatible with the VoIP QoS requirements.

Sets of experiments have been also carried out in order to compare IAX (see [20] and following) and SIP [21] data channel performance differences, shown in Figure 8. No significant difference has been noted between the two protocols, even in the presence of background traffic to fill the available bandwidth. It should be observed that the results do not include traffic multiplexing and/or SIP signalling channel synchronization. In the presence of interfering UDP traffic, the sum of queuing and propagation delays produce a detrimental effect on call quality, as shown in Table II, which summarizes the MOS rating of the sample of users with different 
codecs and UDP interfering traffic flowing at different bit rates (Table II, first row, column 3rd-7th). As shown in Table II, with a shared satellite capacity of $1.4 \mathrm{Mbit} / \mathrm{s}$, the voice call cannot be initiated (marked as $\mathrm{X}$ in the table) at signalling level with an interfering traffic saturating the link. With $1.2 \mathrm{Mbit} / \mathrm{s}$, the SIP protocol connects the terminals but the voice signals are impaired (marked as LAGGED in the table). With $1.1 \mathrm{Mbit} / \mathrm{s}$ of UDP traffic and less, the call quality improves with moderate differences among the selected codec (i.e. GSM, G.711a, and iLBC).

It is also worth noting that, as reported in the first column of Table II, the standard MOS values for each tested codec represent an upper bound that is only partially approached when the bit rate of the UDP interfering traffic is set to $0.35 \mathrm{Mbit} / \mathrm{s}$. This is particularly true for G.711a and iLBC, whereas the case of GSM shows a significant performance gap in terms of MOS (measured to 3 ) with respect to the standard value (set to $3.7 \div 3.9$ ).

\subsection{WICHMO: Description of the experiment}

WICHMO considers a hybrid network with a Wi-Fi link at the network edge and a satellite link somewhere in the network core. Examples of scenarios where this configuration is realistic are ships or airplanes where Internet access on board is provided through a Wi-Fi access point and a satellite link with a geostationary satellite [22]. The serialization of terrestrial and satellite wireless links is problematic from the point of view of a number of applications, be they based on video streaming, interactive audio, or TCP. The reason is the combination of high latency, caused by the geostationary satellite link, and frequent, correlated packet losses caused by the local wireless terrestrial link [23].

In WICHMO we concentrate on frame error models designed to investigate the performance of TCP-based applications on such hybrid networks. Since TCP interprets packet loss as a sign of congestion, thus slowing its pace to avoid worsening the network conditions, frame errors due to corruption cause decreased throughput, and this effect is worsened by high path latency. Various techniques exist that tackle this problem [24], but no definitive answers yet.

While errors are rare on the satellite segment, an error model is required for the terrestrial segment. A complete packet error model for the Wi-Fi link requires three levels of operation: a frame error model at the raw channel level, an implementation of the Wi-Fi $A R Q$ (automatic retransmission) mechanism, and an implementation of the vendor-dependent speed switching mechanism. The first level gives a statistical representation of the frame error process on the raw channel due to either bad preamble acquisition or bad CRC due to corrupted bits in the MAC protocol data unit. The second level implements ARQ as defined in the IEEE 802.11 standard, whereas a sender retransmits a frame up to a configurable number of times-typically 7 - or until it receives an acknowledgement. The third level implements an adaptive coding and modulation scheme (ACM), by which the sender may switch to a different transmission speed when it perceives a change of the channel conditions.

WICHMO tackles the first two levels by choosing a statistical frame error model that is adequate for TCP on a hybrid channel with fixed-speed Wi-Fi (no ACM). To this end, we run a simulation by using real traces of frame errors on a Wi-Fi channel; we then rerun the simulation by using synthetic traces of frame errors produced by commonly used models, and compare the results. WICHMO additionally considers the third level (i.e. ACM) on a Wi-Fi-only connection: integration of the latter results with the former ones is a matter of future research. 
4.3.1. Hardware/software used. We obtained real traces of the packet losses both at the CNRISTI Institute in Pisa (IT) and at the Engineering School at the Universitad Autónoma de Barcelona (ES).

Pisa experiments were concerned with the frame error process with ARQ but no ACM. In Pisa, we connected a Wi-Fi ad hoc network to the Skyplex satellite system to test the feasibility of the experiment, which we did successfully. We then considered the terrestrial and satellite segments separately, in order to obtain sets of separate parameters.

As far as the satellite link is concerned, we considered a $2 \mathrm{Mbit} / \mathrm{s}$ bandwidth with a latency of $300 \mathrm{~ms}$, a transmit buffer sized as the bandwidth-delay product, which is $150 \mathrm{kB},{ }^{* *}$ and a Bernoullian channel with an FER of $10^{-5}$, which in our case is essentially the same as errorless [25].

As far as the Wi-Fi link is concerned, we used two laptops equipped with a Debian operating system and PCMCIA Wi-Fi interfaces configured in ad hoc mode. Measurements were carried out in an office where thin concrete walls delimit the rooms. The transmitter sent a flow of 1000byte unicast frames spaced by $5 \mathrm{~ms}$, at a fixed rate of $11 \mathrm{Mbit} / \mathrm{s}$, with fragmentation and retransmission disabled. The receiver checked the sequence number inside the frames and kept a trace of the lost ones. Since the channel occupancy of a frame is about $1.2 \mathrm{~ms}$, this kind of measure traces the wireless indoor channel conditions quite accurately.

Barcelona experiments were concerned with ACM performance, so both retransmissions (ARQ) and adaptation to channel (ACM) were enabled. Measurements were carried out at a floor with corridors and classrooms. We measured the packet losses for different channel states, each state being defined as a range of the signal to noise ratio (SNR). The dynamics of the slowly changing signal strength has been modelled in the literature as a memory-less Markov chain [26]. Within each state, we measured the packet loss rate by using an Intel Pro Wireless $2200 \mathrm{BG}$ card. We measured the channel in several representative scenarios in order to obtain a general indoor channel model.

4.3.2. Results: comparison of traces with commonly used packet error models. Results relative to frame errors and ARQ without ACM were obtained in Pisa by simulating the hybrid network on ns-2. Results obtained by feeding the simulator with real frame error traces measured on the Wi-Fi link were then compared with results obtained by feeding the simulator with synthetic traces based on two different frame error models, in order to choose the most useful Wi-Fi frame error model for TCP over hybrid networks.

Results relative to ACM, which were obtained in Barcelona, show the influence of ACM over Wi-Fi performance: integration of these results in the hybrid scenario will be the subject of future research.

TCP performance on synthetic versus measured packet loss: The most common frame error model used for Wi-Fi channels is the Bernoullian process (sometimes called a Poisson process), which is defined by a single parameter $p$, the probability that a frame is not received correctly. A slightly more complex model defines the channel as being in one of two states, namely a good state, where all frames are successfully received, and a bad state, where all frames are lost because of corruption; two parameters are required, which are the probabilities of switching from one state to the other. We refer to this good-bad model as a bistable model. A simple

\footnotetext{
*** Buffer size $[\mathrm{kB}]=2 \times(2000 \mathrm{~kb} / \mathrm{s} \times 0.3 \mathrm{~s}) / 8$ bits $/$ byte.
} 


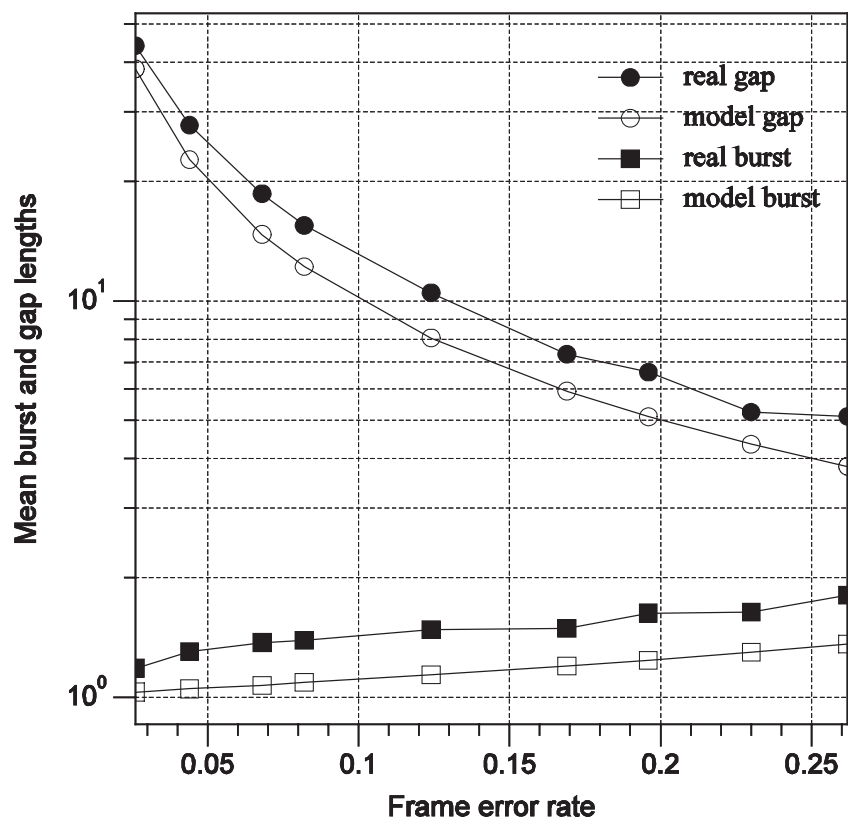

Figure 9. Mean burst and gap length distributions for the measured data and for a Bernoullian channel with the same mean frame error rate.

statistic highlighting the difference between the Bernoulli model and the bistable model is the mean length of bursts and gaps, i.e. of uninterrupted sequences of bad and good frames, respectively. Apart from simplicity, these statistics are significant in the context of Pisa experiments: in fact, TCP increases its transmission speed as long as no segment is lost (which depends on the gap length) and may significantly reduce it if many consecutive packets are lost (an occurrence depending on burst length).

Both the Bernoulli and the bistable models have geometrically decaying correlations. However, the frame error traces we measured exhibit statistics different from both aforementioned models, which is the main ground for this investigation. Figure 9 shows the mean lengths of bursts and gaps versus FER as observed in our measurements.

To simulate the behavior of TCP in hybrid networks made up of a satellite link and a Wi-Fi link, without ACM, we used the ns-2 simulator with Ttem (http://wnet.isti.cnr.it/software/ ttem.html), a purposely written $n s-2$ error model that reads a trace of the channel state, applies the IEEE 802.11 ARQ algorithm, and discards packets that cannot be received after a given number of retries, seven in our case. By using Ttem, we could measure what the performance of $\mathrm{Wi}-\mathrm{Fi}$ is on the channel for which we have real frame error traces and compare it with channels where synthetic frame error traces are used.

We run 10 instances of a single TCP connection for $2000 \mathrm{~s}$ on the simulated hybrid network [25]. In order to compare the qualities of error models, we plot the single-connection TCP goodput versus mean FER on the raw channel (i.e. frame errors before ARQ); we do so by using synthetic traces generated by the model, and compare the resulting graph with the one obtained using real traces. In other words, we compare the goodput obtained when frame errors are 
Table III. Traces used in the simulation experiment.

(a) Bernoulli process having the same frame error rate as the real traces

(b) Bernoulli process having the same mean burst length as the real traces

(c) Bernoulli process having the same mean gap length as the real traces

(d) Bistable process having the same frame error rate, mean burst length and mean gap length as The real traces

(e) The real traces

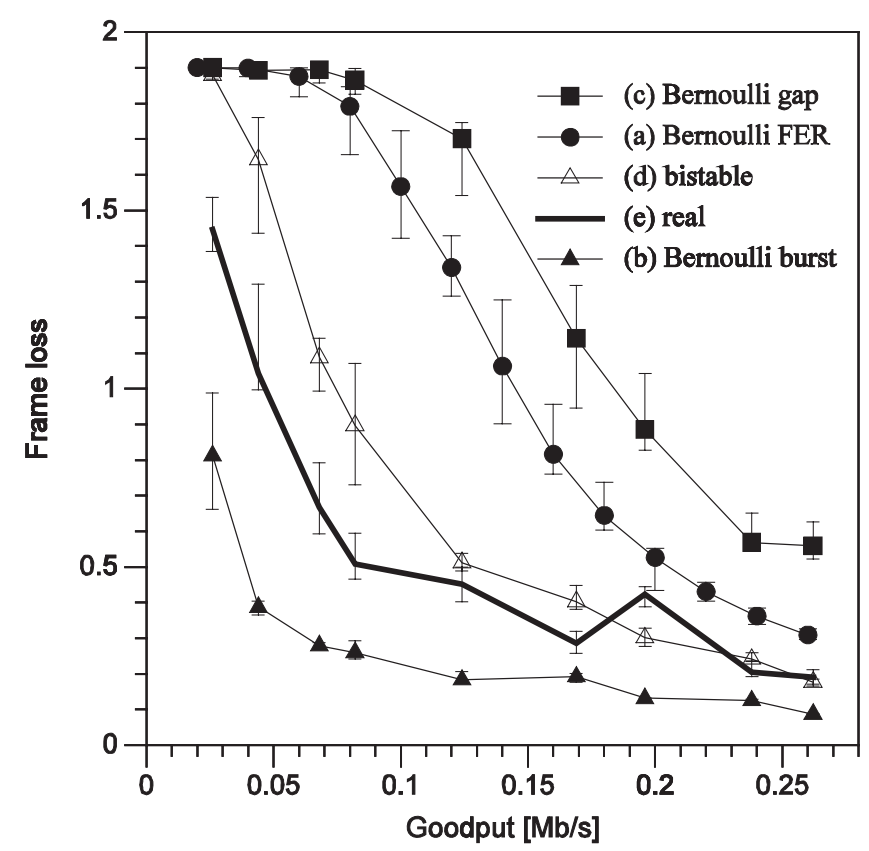

Figure 10. TCP goodput versus frame error rate of real traces for different error models. The error bars indicate the minimum, mean, and maximum goodput values.

generated according to Bernoulli and bistable models with the goodput obtained when frame errors are those measured in the real indoor scenario. Taking the steady-state TCP singleconnection goodput as a performance measure, we see how well different statistical models fit the measured frame error traces. We consider synthetic traces generated by four different frame error models in addition to the real traces, as shown in Table III, and plot the goodput computed using the different models in Figure 10.

To understand how the three models (a), (b), and (c) in Table III differ, consider that, when using a Bernoulli process as the frame error model, it is necessary to tune a single parameter to fit the model to observed data; that is, one should choose a significant statistical parameter of the observed data and use its value to generate the Bernoulli process. The choice of such a parameter is not obvious. In our case, the simplest choice of a parameter is the mean FER. However, there are some good reasons why choosing a different parameter could be wiser. One candidate as an alternative to the FER is the mean burst length, an important parameter with 
respect to ARQ performance: the longer the error bursts, the higher the probability that ARQ cannot recover a lost frame. Another candidate is the mean gap length, which is related to TCP performance. As observed in [27], TCP performance is higher for higher burstiness of the segment error process, because the congestion window has a higher probability of becoming big if gaps are long. In fact, the measured error process has both longer bursts and longer gaps than a Bernoulli process with the same FER.

The main result of the Pisa experiment is the plot in Figure 10, where the goodput of TCP on the hybrid network is plotted versus the FER of the real traces of the Wi-Fi segment. The error bars indicate the minimum, mean and maximum goodput values over the 10 runs. A model should be considered to perform well if the relative goodput line graph resembles the goodput line (e), which is obtained from real traces. Figure 10 shows that (a), the simplest frame error process, does not adequately model TCP's goodput on the hybrid network: while the curve (e) is convex, (a) is not. Using the mean burst length as the parameter of interest produces a behavior resembling the one of the real traces, as it can be seen by comparing cases (b) and (e), but the values are very different. As mentioned above, this observation is a hint that the behavior of ARQ is the main cause influencing the TCP goodput. On the other hand, the gap length appears to be much less important; in fact, using it as the parameter for tuning the Bernoulli process does not yield satisfactory results: the trace for the (c) case is the one farthest from (e), which is the reference curve. One reason could be that, as shown in Figure 9, a Bernoulli process with the same mean gap length as the real traces has a lower FER than the real traces. While not perfectly satisfactory, case (d) is the most similar to (e): the bistable model, which uses two parameters instead of one, best fits the chosen performance measure and is our recommended choice.

Adaptive coding and modulation effect on the hybrid channel: In the Barcelona experiment, we analyzed the third level of a packet error model, i.e. the effect of the adaptive modulation and coding (ACM) scheme of the WLAN card on performance, this time independently of the satellite link. All members of the IEEE 802.11 WLAN family $(802.11 \mathrm{a} / \mathrm{b} / \mathrm{g})$ provide multi-rate capabilities. To achieve a high performance under varying conditions, these devices dynamically adapt their transmission rate to the channel conditions. While this rate adaptation algorithm is a critical component in the overall system performance and it also is a critical component of the accuracy of a packet error model, only very few algorithms have been published and the implementation issues associated with these mechanisms are hardly publicly available.

The experiment we have set up consists of measuring the packet loss for different channel states, each state defined as a range of SNR. The dynamics of this slow term signal loss have been modeled in the literature as a memory-less Markov chain [26]. Within each state, we measured the packet loss rate. We focused on an indoor environment and we measured the channel in several representative scenarios in order to obtain a general indoor model.

Figure 11 shows the measured nested channel states of received power (black line) and packet loss (gray line), respectively. Note that during the snapshot shown the channel only experienced the states under shadow conditions, i.e. 2 and 3. Figure 12 shows the comparison of mean length of error bursts and gaps both without applying rate adaptation and applying two different rate adaptation algorithm for IEEE802.11 WLANs, i.e. automatic rate fallback (ARF) and LD-ARF.

ARF [28] was the first-rate adaptation algorithm to be published. In the ARF algorithm, a discrete set of data rates are used. If the ACK frames for a given number $N_{\text {down }}$ (equals to one if the rate has just been increased, otherwise equals to two, as specified in [28]) consecutive data 


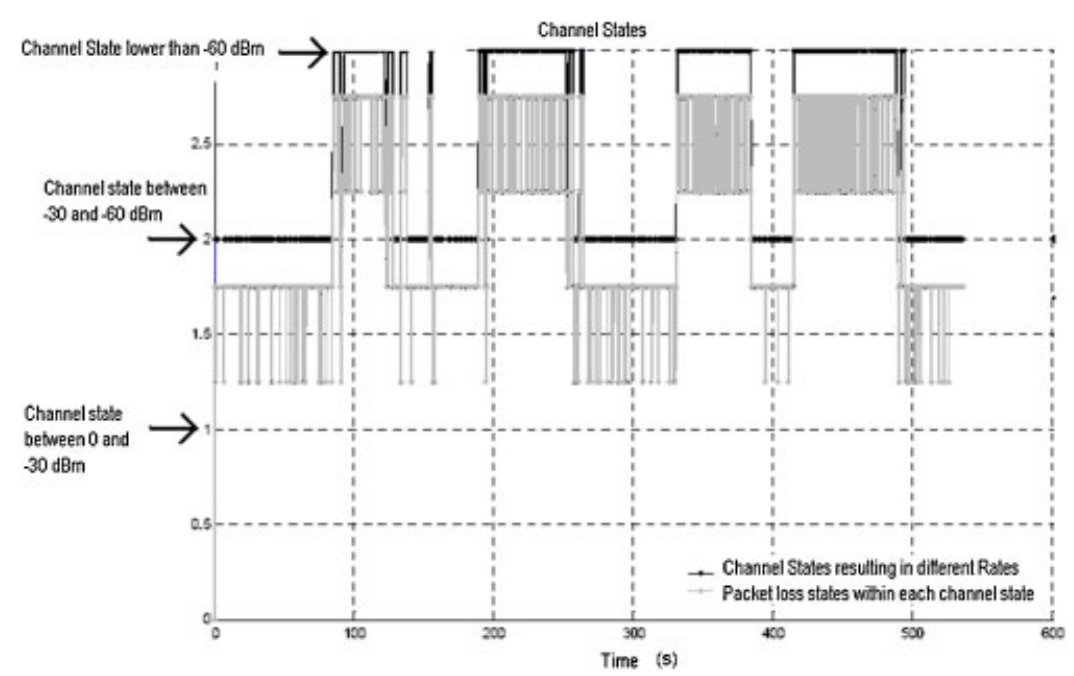

Figure 11. SNR states and packet loss within each state. Channels III, II, and I correspond to SNR lower than $-60 \mathrm{dBm}$, between -60 and -30 , and above $-30 \mathrm{dBm}$, respectively.


Figure 12. Mean error and successful burst length probability when ARF and LD-ARF are considered compared to the case where no rate adaptation is applied.

packets are not received by the sender, then the sender drops the transmission rate to the next lower data rate and a timer is activated (rate-up timer). If $N_{\text {up }}$ (equals to 10 as given in [28]) consecutive ACK frames are received or the timer expires, then the transmission rate is raised to the next higher data rate and the timer is stopped. When the rate is increased, the first transmission (also called probing frame) after the rate increase must be successful or else the rate is immediately decreased and the timer is started again. Packet collisions are the main obstacle to ARF performance since, when a collision occurs, ARF decreases the data rate as it cannot distinguish between a packet loss due to link state and collision. However, the standard MAC 
protocol provides no mechanism to differentiate between the two types of losses and therefore both the MAC layer protocol and the ARF algorithm need to be modified.

Aside from ARF, other rate adaptation algorithms have been proposed in the literature, based on different concepts. In [26] an algorithm called loss-differentiation ARF (LD-ARF) is proposed. This algorithm solves the collision problem by differentiating losses through MAC layer modifications. We have chosen this algorithm since the increase in throughput is significant compared to the ARF.

The comparison of mean error burst and gap length probabilities in three cases is depicted in Figure 12: without applying rate adaptation, and applying the two different algorithms. It can be seen that not only the average burst length duration decreases significantly, but also the statistical behavior changes with respect to not considering rate adaptation.

It is interesting to point out the non-intuitive results that are shown. When rate adaptation is applied, the rate is increased after having received a pre-defined number of successful transmissions, whereas it is decreased by a pre-defined number of unsuccessful transmissions or measured performance threshold. This means that adaptation to bad channel conditions results into a lower bit rate and, therefore, long error bursts. On the other hand, adaptation to good channel conditions results in high bit rate thus implying short successful burst duration. Moreover, the better the performance of the adaptation algorithm, the shorter the successful bursts. Clearly, shorter bursts with rate adaptation imply higher throughput than longer bursts without rate adaptation. The effect on throughput can be easily quantified but it requires different cases and scenarios to be defined and cannot be shown here for space limitations.

\section{THE INTEGRATED TESTBEDS: UCIT AND EGGS}

\subsection{UCIT: a tool for performance evaluation}

The challenges exhibited by satellite communications, in terms of large bandwidth-delay product and link errors, have been thoroughly explored over the last decade. In addition, the extension of this environment including also wired and wireless links, has fostered the scientific community toward the definition of a large number of transport protocols and architecture solutions tailored to this larger scenario. Here, we limit ourselves to cite TCP Hybla [29] and TCP-Westwood [6] as transport protocols, even if this could be further extended. An alternative approach, widely adopted in satellite environments, is represented by 'accelerators' or performance enhancing proxies (PEPs) [30], which basically rely on the introduction of intermediate agents at transport layer. In a future perspective, the delay/disruptive tolerant networking (DTN) architecture [31] may represent an interesting solution not only for deep space communications, but also for the most challenging satellite networks.

Given this complex context, it is paramount to design and set up effective tools to comparatively evaluate the proposed solutions in a controlled testing environment. For this purpose, CNIT and the University of Bologna (UoB) decided to integrate their assets and expertise to realize the UCIT common testbed platform for performance evaluation at the transport layer. Here, we focus on the description of this testbed, referring, whenever necessary, to the configuration recently used to carry out a series of joint experiments in cooperation with the Network Research Lab at UCLA, Computer Science Department. 




Figure 13. Testbed logical layout.

5.1.1. Testbed overview. The testbed logical layout, sketched in Figure 13, aims at reproducing a heterogeneous network, where satellite connections have to compete for network resources with wired connections.

Of course, it is also possible to study a pure satellite environment by simply disabling background terrestrial components. Satellite connections are composed of wired legs and a final satellite link, while TCP background traffic is only present in the entirely wired paths. All TCP variants available in the Linux kernel are supported as well as the FreeBSD implementation of Westwood (directly provided by UCLA). Satellite and wired connections share the Router $1-$ Router 2 link, whose bandwidth can be limited in order to study congestion effects. The satellite link can be realized either by emulation or by means of any real satellite system, as done in the joint UoB-CNIT-UCLA measurement campaign, where the Skyplex platform was exploited. Finally, the testbed allows the user to assess the performance of alternative solutions, based on satellite accelerators or on the DTN architecture.

5.1.2. Testbed components. To describe in more details the testbed components, let us refer to the much more complex physical layout of the testbed, in the configuration used during the recent joint experiments' campaign (Figure 14). The following components can be distinguished:

- a testbed controller, located at UoB;

- the UoB TATPA testbed (testbed on advanced transport protocols and architecture) [32], temporarily relocated in Genoa,

- a TCP satellite receiver (one Linux PC) in Naples and the CNIT Skyplex GEO satellite platform [3], which links the testbed core with the satellite receiver. We will examine the main components separately.

5.1.3. Testbed controller. The controller, connected to the TATPA core via a virtual private network, allows the user a ubiquitous remote control of the testbed via a standard web browser. A dedicated PC hosts the web server and the control software engine, developed in PHP and based on a MySQL database. The aim of the TATPA web interfaces and management control system is to facilitate the shared use of the testbed. In particular, they allowed us to hide the software and hardware complexity related to the configuration, synchronization, and utilization 




Figure 14. Testbed physical layout.

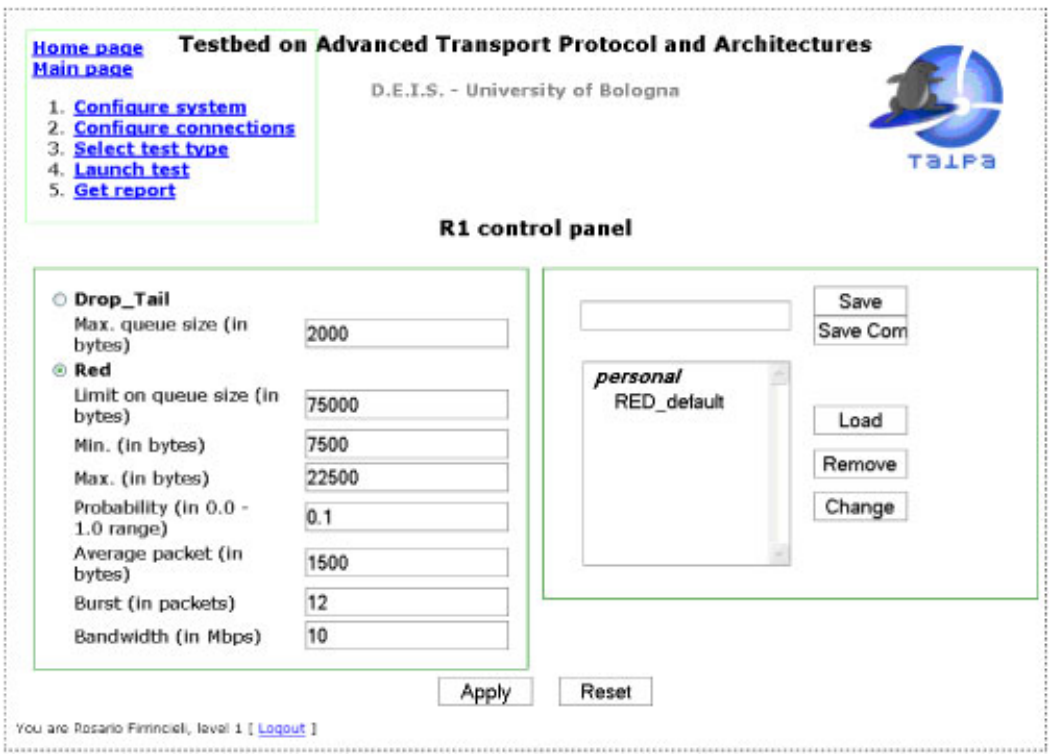

Figure 15. TATPA web interface: configuration of Router 1 settings. 
of all testbed elements. Moreover, the web controller provides an increased level of security, as users cannot access testbed components directly, and greatly speeds up both test execution and result collection.

As an example, the configuration page of Router 1 is given in Figure 15. Analogous interfaces are available for all the testbed entities. They can be opened by simply double clicking on entity icons on the main configuration page.

5.1.4. The TATPA core. The TATPA core consists of several PCs running the Linux operating system (kernel 2.6.20), and a PC running FreeBSD, which is directly maintained by UCLA. Multi-TCP [33] on Linux senders implements the full version of TCP Hybla (including packet spacing and Hoe's initial ssthresh estimation) and introduces powerful log functions, essential for an in-depth analysis of results. In particular, they allow to retrieve meaningful indications about the TCP dynamics, by capturing the values of the internal TCP variables (e.g. congestion window $c w n d$, slow start threshold ssthresh, and retransmission timeout $R T O$ ) It is worth noting that the internal TCP variables are extracted directly from the kernel core, since common traffic analysis tools (e.g. tcpdump) cannot derive values of TCP variables.

Router 1 can follow either a drop tail policy or a random early detection policy (see Figure 15). The PEPsal package [34] is installed on the Router 2 to evaluate the performance of satellite accelerators. Finally, to study DTN architectures, DTN agents have been mounted on both the satellite sender and receiver, as well as on the Router 2. DTN performance can be evaluated by means of DTNperf application [35].

5.1.5. Data analysis. Owing to the features offered by the aforementioned three components of the testbed, a user is allowed to specify and run tests aimed at assessing the performance of TCP/ UDP traffic flows and, alternatively, of DTN architecture applied over heterogeneous satellite networks. The tests consist in transferring data for a time duration specified by the user at the beginning of the trials. Transfer of data is managed by the testbed core transparently to the user and performed by means of either Iperf or DTNperf tools, in dependence on the specific protocol architecture under investigation. After collecting results through the integrated testbed, they have to be processed and carefully examined, in order to draw general conclusions not only about the effectiveness of different solutions, but also about the mechanisms that lead to different performance. To this end, the capacity of analyzing also the internal TCP variables is instrumental.

To show the features offered by the integrated testbed, let us consider the example given in Figure 16, which refers to a TCP Hybla file transfer of 200 s, on the CNIT-Skyplex channel, in absence of background traffic. The time sequence of the segments sent are given in the figure, together with the cwnd and the ssthresh dynamics. All the data were collected by the $\log$ functions of the MultiTCP package and, successively, made available by the web interface.

From Figure 16 meaningful information about the dynamics of this TCP variant under study can be inferred. Let us start from the analysis of $c w n d$, which reflects the enhanced congestion control algorithm introduced by Hybla. Aiming at removing the penalization due to long RTTs, it allows a fast opening of the $c w n d$ even on a long propagation delay GEO satellite channel (RTT larger than $600 \mathrm{~ms}$ ). Losses determine $c w n d$ reduction according to the Linux rate-halving algorithm, which instead of halving the $c w n d$, gradually reduces it during the fast recovery phase. TCP retransmissions during the recovery phase are indicated by Tx segments with a sequence number lower than previously transmitted segments. The relatively long burst of 


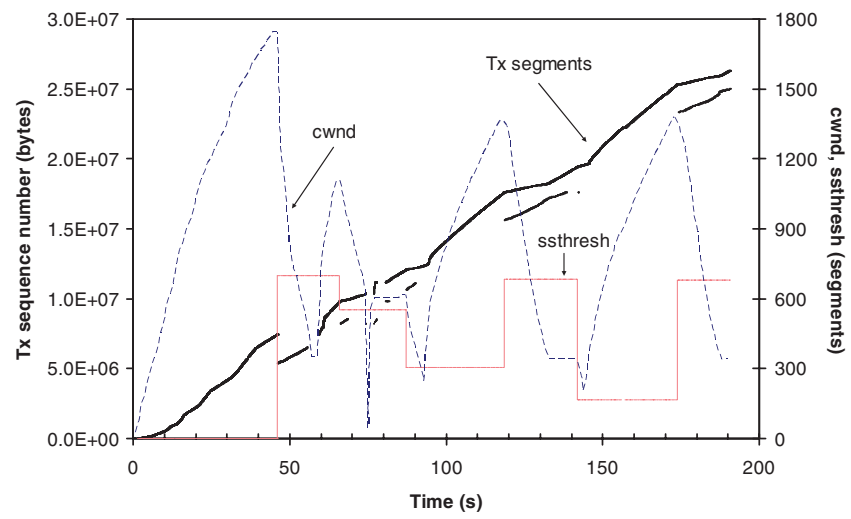

Figure 16. Example of analysis: segments sent, cwnd, and ssthresh curves. Hybla, CNIT Skyplex channel, no background traffic.

retransmissions present in the Tx time sequence are likely indicators of buffer overflows. At the end of loss recovery phase, the $c w n d$ rapidly increases, thanks to the Hybla enhanced congestion control, until new loss events are detected. Hybla ssthresh dynamics is also reported in Figure 16. In correspondence of loss detection events, ssthresh is set to half of packets in flight. After the recovery phase conclusion, cwnd is increased following the Hybla slow start or congestion avoidance laws, depending on the relative values of cwnd and ssthresh.

The UCIT integrated testbed is an important result of the integration activities carried out in SatNEx. The integration of assets of different partners was instrumental to obtain a powerful tool for performance evaluation of transport protocols and architecture. It was used in two joint UoB-CNIT-UCLA measurement campaigns. An excerpt of the results obtained in the first campaign is reported in [36].

\subsection{EGGS}

5.2.1. Description of the experiment. Remote measurement operations, habitat, and environmental control tasks in interplanetary scenarios give raise to challenging issues, in particular in the design of communication protocols. Many peculiarities characterize interplanetary networks: large propagation delay, multi-path fading, solar wind, and other hostile radiofrequency conditions that impact on the channel performance. Additionally, because of celestial motion, the line of sight is not always available. The results are channel disconnections (expected or not), and high bit error rates (ranging from $10^{-3}$ to $10^{-2}$ ) [37]. For these reasons TCP/IP is not the best candidate. Alternative solutions are DTN and protocol architectures proposed by the Consultative Committee for Space Data Systems protocols [38], or customized flavors of TCP. Given such a wide choice of possibilities, implementing each single protocol is not tractable; especially if we consider that some of these proposals are still under development, hence permanently evolving. Testing over real testbeds belongs to the future. An experimental tool, which makes possible to use protocols already implemented, without the costs and complexity of real testbeds is thus strongly needed. In particular, the EGGS experiment is aimed at evaluating the performance of adequate protocol architectures, suited to transport data over interplanetary networks, by addressing most of the design challenges that may arise in this harsh 
environment. To this end, a distributed testbed, composed of remote emulation platforms, has been used in order to develop a thorough analysis of all the aspects that require to be considered from the implementation point of view. In more detail, attention was mainly paid to the networking issues by pointing out the advantages offered by the design of such a prototype. The overall scenario is composed of:

- an Earth station responsible for collecting data originated by a remote sensor network;

- a sensor network [39] located on a remote planet surface;

- a satellite orbiting around the remote planet, serving as relay point between the planet and the Earth.

The presence of different satellite links together with the sensor network makes the use of a single platform impractical. The integration of different emulation tools is strongly required in order, on the one hand, to distribute the whole network complexity among several components and, on the other hand, to control the granularity aimed at characterizing the peculiarities of each network portion. It is possible to individuate two kinds of links:

- a proximity link, established from/to the remote planet and the satellite orbiting around;

- a long-haul link, providing the data communication over the deep space path;

- besides, the necessity to dedicate specific tools for each considered interplanetary link (proximity and long haul) was motivated by the very different characteristics in terms of delay, bandwidth, and packet loss patterns.

5.2.2. Hardware/software used. The integrated testbed is characterized by a long-haul satellite link emulator (called ACE) [40], a sensor testbed (SENS), and a proximity satellite link emulator (DUMMYNET) [41]. In more detail, ACE was located by the University of Genoa, SENS by ISTI-CNR institute, and DUMMYNET was available at GET/ENST (Groupe des Ecoles de Télécommunications-Ecole Nationale Supérieure des Télécommunications) premises in Toulouse. The tools were virtually integrated by means of 2 IP tunnels set between SENS and ACE, and between ACE and DUMMYNET, respectively. Different tools are used for the long haul and proximity links because the requirements inferred by the environments are not the same.

The long-haul link: Concerning ACE, its basic aim is to emulate a network environment composed of a set of Earth stations that communicate through a satellite link. Its extension toward interplanetary communications is straightforward. It can emulate the data communication among an Earth station, responsible for gathering data arriving from the remote sensor network, and a satellite platform orbiting around the remote planet. Under this view, it is possible to think of these terminal agents as PCs connected to each other by means of an emulated satellite link, working as follows. The whole system is composed of three devices, hosting a Linux O. S, whose role is to perform the basic functionalities of data forwarding and to fully characterize the transmission channel. For this purpose, the emulation task is performed at the data link layer, in order to take into account frame formats, channel coding schemes, and proper physical layer characteristics, such as propagation delay, channel bandwidth availability, and statistical behavior of the satellite link (e.g. in terms of bit error ratios and channel modelling).

The proximity link: As far as the proximity link emulation is concerned, it takes the Dummynet Free BSD kernel extension as reference. It allows tuning the propagation delay, the 
bandwidth availability, and the link reliability (e.g. random, uniform loss of packets) through the application of proper schemes and policies implemented at the IP layer.

The sensor network: The sensor testbed consists of a set of Micaz motes from Crossbow Inc. [42]. They are equipped with an $8 \mathrm{MHz}$ microcontroller, an IEEE 802.15.4-compliant radio [43] and transducers to sample light, temperature, acceleration, magnetism, and audio. The embedded software implements a distributed database management system [44] where relational algebra operators, including selection, projections, and joins can be carried out on the nodes and interconnected via data stream channels. Users draw the layout of a query graphically and interactively via a GUI application. ${ }^{\dagger \dagger}$ The query layout concisely represents all data sampling, data processing, and data transfer activities to fulfil on each of the network nodes. The GUI interacts with the remote sink via a TCP connection for both sending commands and receiving sensor data. The experimentation core is based on running queries with a variable number of sampling nodes (during the initial tests, it ranges from one to three) and applying different sampling rates (from 100-2000 ms). The measurements, which typically involve light variations, are properly processed by the sensor network and encoded to allow their transmission to the sink node, which, in turn, relays them to the GUI.

The three components (ACE, DUMMYNET, and SENS) described before are located at different premises (Genoa, Toulouse, and Pisa). In order to link the three sites, a VPN topology is established with the advantage to make the use of a private addressing plan possible. The drawback of this distributed approach is the natural delay introduced by the Internet: it has to be taken into account. A Perl script repeatedly measuring the RTT helps to adjust the delay as needed.

5.2.3. Emulation results. Two scenarios are considered: (a) full-TCP and (b) DTN/TCP. In the former, the sensor network GUI is located on the Earth and a TCP connection is established with the sensor network sink. The TCP connection therefore goes over the long-haul link with the expected limitations of TCP.

In the DTN/TCP scenario, the sensor network is connected to the orbiter via a TCP connection (proximity link). A DTN/TCP proxy in the orbiter translates sensor data sent from the sink into DTN bundles and forwards them via UDP to the Earth. The DTN/TCP proxy is written in Perl. The DTN stack is the reference implementation available from the DTN Research Group [45]. The sampling rate of the sensor network for the DTN scenario is set to 10 measures per second while it is twice that value for the full-TCP scenario. A measure is approximately 25 bytes. The current implementation of the DTN stack has difficulties to sustain high rates and issues a threading runtime error. Finally, all network links are constrained to a throughput of $64 \mathrm{kbit} / \mathrm{s}$. The propagation delay for the long haul link ranges from $125 \mathrm{~ms}$ to $200 \mathrm{~s}$. The proximity link has a propagation delay of $40 \mathrm{~s}$.

Measures are collected in the Earth station (i.e. in Genoa), being tcpdump used to record traffic traces. Currently, bit errors are not taken into account in the experiment, although they can be generated by the two link emulators if needed.

The full-TCP scenario: As far as the full TCP scenario is concerned, particular attention has been paid to the impact of large latencies on the transmission protocol. To better understand

\footnotetext{
${ }^{\dagger}$ A graphical user interface (GUI) is an application that uses the standard Java components GUI component set, Swing, and is deployed to the desktop. Like all Java code, Java GUI applications simplify the task of creating desktop applications because they can be run on any platform.
} 


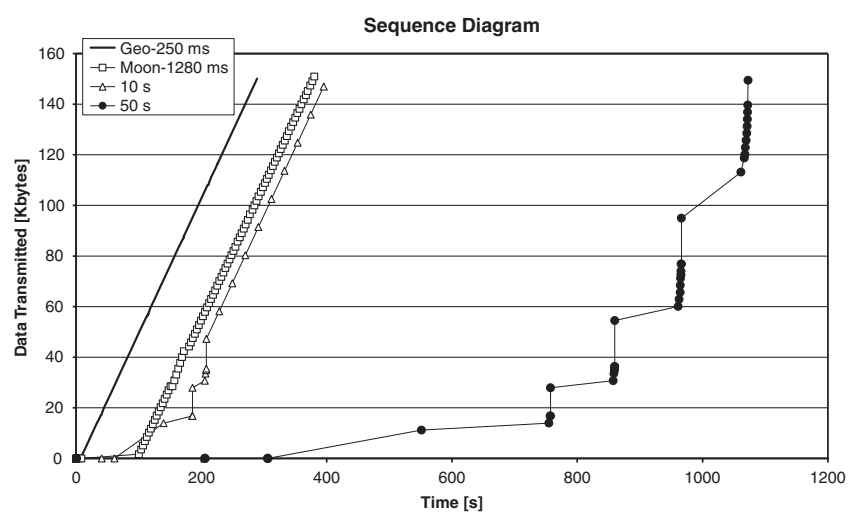

Figure 17. Diagram sequence for the four investigated scenarios.

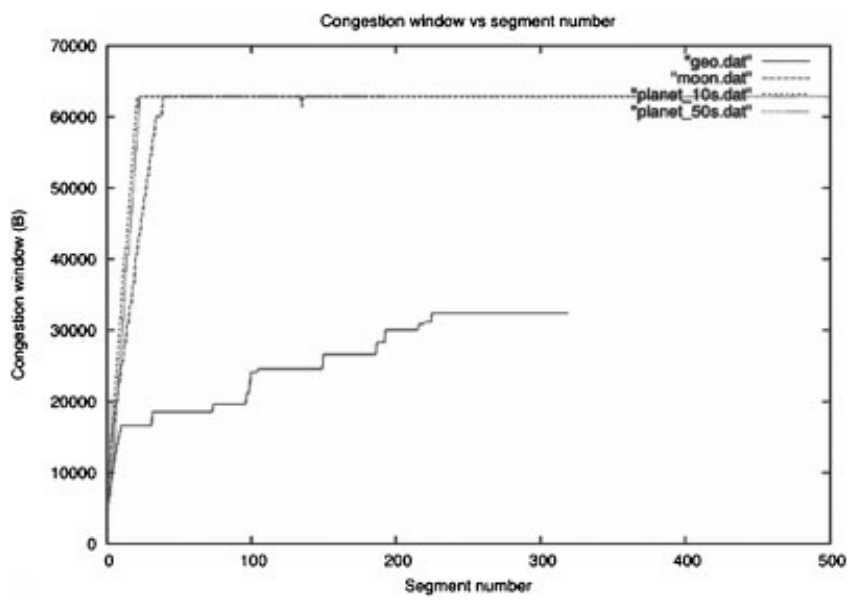

Figure 18. Congestion window diagram over TCP segments.

this aspect, and hence to assess the operation of the proposed emulation platform, different propagation delays have been considered for the long haul link (250 and $1280 \mathrm{~ms}, 10,50$, and $200 \mathrm{~s})$. We first tested the behavior of TCP when the largest propagation delay ( $200 \mathrm{~s})$ is set. This trial showed, as expected, the unfeasibility of using TCP protocols over 'very long' networks, due to the TCP timers and related algorithms tuned to the more common terrestrial path delays. To tackle these problems, TCP parameters have been tuned accordingly to the environment peculiarities. Trials with delays equal to 250 and $1280 \mathrm{~ms}, 10$ and $50 \mathrm{~s}$ have been successfully completed, while the case of $200 \mathrm{~s}$ still experienced some hazards, because of the limits of the TCP back-off algorithm triggered during timeout expirations.

Figures 17 and 18 ('Geo' and 'Moon' configurations refer to delays of 250 and $1280 \mathrm{~ms}$, respectively) show the behavior of instantaneous packet transmissions and congestion window over the elapsed time and the TCP segment number, respectively. These graphs confirm that the 


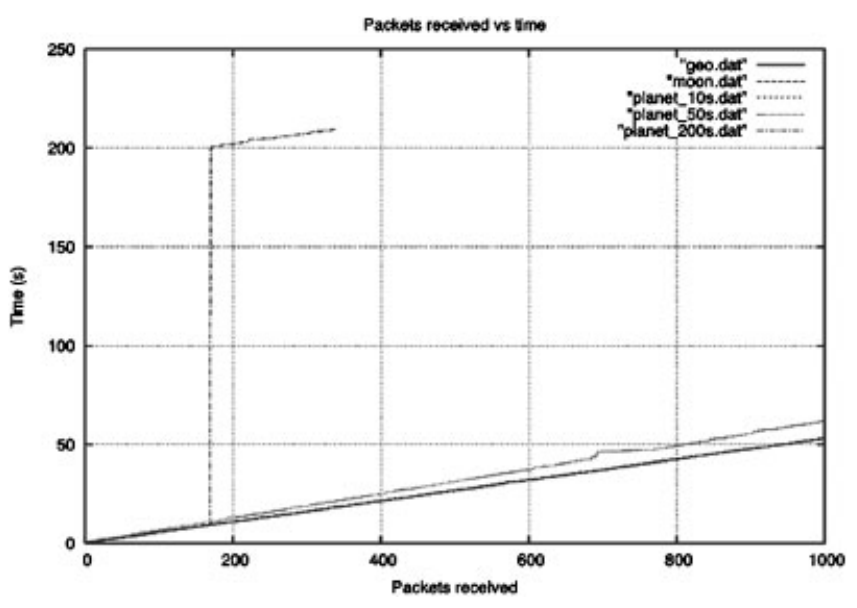

Figure 19. Received datagrams versus time for the DTN/TCP trial.

long haul link propagation delay impacts the dynamics of data transfer changes. In particular, Figure 17 demonstrates that for a $250 \mathrm{~ms}$ delay, the transmission of TCP segments follows a quasi-linear law. On the other hand, as delay increases, the time interval required to transmit the same amount of data gets larger. In particular, it is possible to see that for delays of $1280 \mathrm{~ms}$ and $10 \mathrm{~s}$, an initial phase where the number of transmitted segments over time keeps low, because of the slow-start phase. Afterwards, the congestion window is almost regular as one may infer from Figure 17, and confirmed by Figure 18. The case of $50 \mathrm{~s}$ deserves more attention. Figure 17 shows that the number of segments sent out over time increases very slowly because of the very large latency. Actually, a comparison with the other configurations shows that the time required to transmit about 150 kbytes is five times larger and nearly equal to $1100 \mathrm{~s}$. Similar considerations still hold also for the congestion window behavior plotted in Figure 18, which shows a stepwise shape in the case of $50 \mathrm{~s}$ delay. This confirms that, in presence of very large bandwidth-delay product networks (as it may happen in interplanetary networks), TCP solutions are not efficient, since the increase in the congestion window is too conservatively ruled by the TCP acknowledgment arrival, which results in long idle times in these scenarios.

Finally, Figures 17 and 18 outline that, in general, even if large latencies are set, the proposed emulation platform is able to match the channel peculiarities. Additional tests have still to be conducted in order to assess the limits of the emulation platform, especially when large delays are used in conjunction with high data rates.

The DTN/TCP scenario: The DTN/TCP experiment deploys the bundle protocol over the long haul link. UDP was selected as a subnetwork, in order to work around issues related to the impairment of conversational protocols over very long networks.

Figure 19 shows the received UDP datagrams with respect to time. The results related to the $200 \mathrm{~s}$ experiment display an interruption in the reception of the UDP flow due to buffer overflow occurred at the long-haul emulator. This event was an indirect consequence of the very large latency that caused long buffer queues on the ACE platform. This behavior is further amplified in long-run simulations that give rise to buffer overruns, as highlighted in this case.

Apart from this critical case, the other configurations with large delay (e.g. 50 and $10 \mathrm{~s}$ ) show the benefits of the DTN architecture implementation. In fact, Figure 19 shows that the number 
of received packets over time linearly increases almost independently of the propagation delay; the curves related to delay configurations of 50 and $10 \mathrm{~s}$, respectively, nearly overlap and, besides, they even keep really close to the delay configuration of $250 \mathrm{~ms}$ (reported as 'geo.dat'). Being congestion control procedures based on feedbacks missing, the main difference exhibited in this case with respect to TCP employment is that bundle rate transmission keeps approximately constant regardless of the investigated environment's physical characteristics.

\section{CONCLUSIONS}

The paper focused on the experimental activities made within the European-funded SatNEx project. They include both measurement campaigns (i.e. VOTOS, VoIP over Satellite, and WICHMO) and realization of integrated testbeds (i.e. UCIT and EGGS). Measurement campaigns provided insights into dynamics of some of the most relevant networking and communication issues in satellite-based scenarios, such as channel modelling, analysis of multimedia applications, and performance study of transport layer protocols. The effect of the DAMA-TDMA access scheme adopted by the Skyplex platform, used in most of the tests, was also investigated. The measurement campaigns proved also useful to validate analytical models (if available) and the accuracy of simulation modules.

Concerning integrated testbeds, their development was dictated by the desire of a more thorough investigation of the issues arising in the development of real satellite systems, including interplanetary scenarios. Their analysis capabilities make them powerful investigation tools, available to the whole satellite community, for studying the dynamics of real satellite systems.

\section{ACKNOWLEDGEMENTS}

Work funded by the European Community in the framework of the FP6 SatNEx NoE project. The project developed in two phases: SatNEx I and II, contract No. 507052 and No. 027393, respectively. The second phase is still in progress.

\section{REFERENCES}

1. Skianis C, Jamalipour A, Fairhurst G, Montpetit M-J, Donadio R. Guest editorial—convergence of Internet and broadcasting systems. IEEE Network 2007; 21(2):4-5.

2. Evans B, Werner M, Lutz E, Bousquet M, Corazza GE, Maral G, Rumeau R, Ferro E. Integration of satellite and terrestrial systems in future multimedia communications. IEEE Wireless Communications 2005; 12(5):72-80.

3. Feltrin E, Weller E, Martin E, Zamani K. Design, implementation and performance analysis of an on board processor-based satellite network. Proceedings of International Conference on Communications (ICC) June 2004; 6:3321-3325.

4. Annese A, Celandroni N, Davoli F, Ferro E, Gotta A. TCP performance measured over wireless integrated networks with high delay-bandwidth products. Proceedings of ASMS, Herrsching, Germany: 2006.

5. Mathis M, Mahdavi J, Floyd S, Romanow A. TCP selective acknowledgement options. RFC 2018, 1996.

6. Grieco LA, Mascolo S. Performance evaluation and comparison of Westwood+, New Reno and Vegas TCP congestion control. ACM Computer Communication Review 2004; 34(2):25-38.

7. Gotta A, Secchi R, Potortì F. An analysis of TCP startup over an experimental DVB-RCS platform. Proceedings of IWSSC, Madrid, Spain. 2006.

8. Handley M, Floyd S, Padhye J, Widmer J. TCP friendly rate control (TFRC): protocol specification. RFC 3448, 2003. 
9. Gotta A, Secchi R, Potortì F. Simulating dynamic bandwidth allocation on satellite links. Proceedings of Valuetools, WNS2 Workshop, Pisa, Italy: 2006.

10. Sathiaseelan A, Fairhurst G. Using quickstart to improve the performance of TFRC-SP over satellite networks. Proceedings of IWSSC, Madrid, Spain: 2006.

11. Floyd S, Allman M, Jain A, Sarolahti P. Quick-start for TCP and IP. RFC 4782, 2007.

12. Debian Linux. Available from:http://www.debian.org.

13. Asterisk VoIP PABX. Available from: http://www.asterisk.org.

14. Trixbox - telephony application platform. Available from: http://www.trixbox.org.

15. AsteriskNOW. Available from: http://www.asterisknow.org.

16. rPath Linux. Available from: http://www.rpath.com.

17. Xlite-SIP SoftPhone. Available from: http://www.counterpath.com.

18. Idefisk-IAX SoftPhone. Available from: http://www.asteriskguru.com/idefisk/free/.

19. WireShark. Available from: http://www.wireshark.org.

20. IAX2: Inter-Asterisk eXchange Version 2 - draft-guy-iax-03. Available from: http://www.ietf.org/internet-drafts/ draft-guy-iax-03.txt.

21. SIP IETF RFCs/WGs. Available from: http://www.sipknowledge.com/SIP_RFC.htm.

22. Davoli F, Ferro E, Mouftah H. Wireless access to the global Internet: mobile radio networks and satellite systems. Guest editorial. International Journal of Communication Systems 2003; 16(1).

23. Barsocchi P. Packet loss in terrestrial wireless and hybrid networks. Ph.D. Thesis, University of Pisa, Italy, 2007.

24. Allman, Dawkinks S, Glover D, Griner J, Henderson T, Heidemann J, Ostermann S, Scott K, Semke J, Touch J, Tran D. Ongoing TCP research related to satellites. IETF, RFC 2760, 2000.

25. Barsocchi P, Oligeri G, Potortì F. Packet loss in TCP hybrid wireless networks. Proceedings of the Advanced Satellite Mobile Systems Conference (ASMS), Herrsching, Germany: 2006.

26. Pang Q, Leung VCM, Liew SC. A rate adaptation algorithm for IEEE 802.11 WLANs based on MAC-layer loss differentiation. Proceedings of the International Conference on Broadband Networks, 2005.

27. Altman E, Avrachenkov K, Barakat C. A stochastic model of TCP/IP with stationary random losses. Computer Communication Review 2000; 30(4):231-242.

28. Kamerman A, Monteban L. WaveLAN-II: a high-performance wireless LAN for the unlicensed band. Bell Labs Technical Journal 1997; 2(3):118-133.

29. Caini C, Firrincieli R. TCP Hybla: a TCP Enhancement for heterogeneous networks. International Journal of Satellite Communications and Networking 2004; 22:547-566.

30. Border J, Kojo M, Griner J, Montenegro G, Shelby Z. Performance enhancing proxies intended to mitigate linkrelated degradations. IETF RFC 3135, 2001.

31. Cerf V, Hooke A, Torgerson L, Durst R, Scott K, Fall K, Weiss H. Delay-tolerant networking architecture. IETF RFC 4838, 2007.

32. TATPA. Available from: https://tatpa.deis.unibo.it.

33. Caini C, Firrincieli R, Lacamera D. A Linux based multi TCP implementation for experimental evaluation of TCP enhancements. Proceedings of SPECTS, 2005.

34. Caini C, Firrincieli R, Lacamera D. PEPsal: A performance enhancing proxy for TCP satellite connections. IEEE Aerospace and Electronic Systems Magazine 2007; 22(8):7-16.

35. Caini C, Cornice P, Firrincieli R, Lacamera D. A DTN approach to satellite communications. IEEE Journal on Selected Areas in Communication 2008; 26(5):820-827.

36. Caini C, Firrincieli R, Lacamera D, De Cola T, Marchese M, Marcondes C, Sanadidi MY, Gerla M. TCP live experiments on a real GEO satellite testbed. Proceedings of IEEE ISCC, Aveiro, Portugal: 2007.

37. Baronti P, Pillai P, Chook V, Chessa S, Gotta A, Hu FY. Wireless sensor networks: A survey on the state of the art and the 802.15.4 and ZigBee Standards. Computer Communications 2007; 30:1655-1695.

38. Akyildiz IF, Akan OB, Chen C, Fang J, Su W. The state of the art in interplanetary internet. IEEE Communications Magazine 2004; 42(7):108-118.

39. Hooke AJ. Towards an interplantetary Internet: a proposed strategy for standardization. Proceedings of Space Operations Conference and World Space Congress, Houston, TX 2002.

40. Marchese M, Perrando M. A packet-switching satellite emulator: a proposal about architecture and implementation. Proceedings of IEEE International Conference on Communications (ICC) 2002.

41. Dummynet. Available from: http://info.iet.unipi.it/ luigi/ip_dummynet/.

42. Crossbow Technology Inc. Available from: http://www.xbow.com.

43. Wireless medium access control (MAC) and physical layer (PHY) specifications for low rate wireless personal area networks (LR-WPANs). IEEE Std. 802.15.4-2003, 2003.

44. Amato $\mathrm{G}$, Baronti $\mathrm{P}$, Chessa $\mathrm{S}$. MaD-WiSe: programming and accessing data in a wireless sensor networks. Proceedings of IEEE Eurocon, 2005.

45. Delay Tolerant Networking Research Group (DTNRG). Available from: http://www.dtnrg.org. 


\section{AUTHORS’ BIOGRAPHIES}

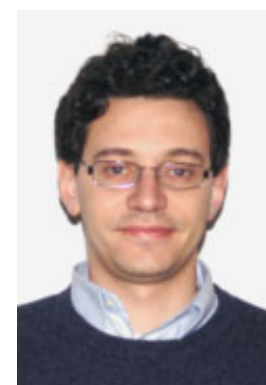

Tomaso de Cola was born in Manosque, France, on April 28, 1977. He received the "Laurea" degree (summa cum laude) in telecommunication engineering from the University of Genoa, Genoa, Italy, in 2001 and the Qualification degree as Professional Engineer in 2002.

From 2002 until 2007, he has worked with the Italian Consortium of Telecommunications (CNIT), University of Genoa Research Unit, as scientist researcher. Since 2008, he has been with the German Aerospace Centre (DLR), where he is involved in different European Projects focusing on different aspects of DVB standards, CCSDS protocols and testbed design. He is co-author of more than 20 papers, including international conferences and journals.

His main research activity concerns: TCP/IP protocols, satellite networks, transport protocols for wireless links, interplanetary networks as well as delay tolerant networks.

\section{Ronga}
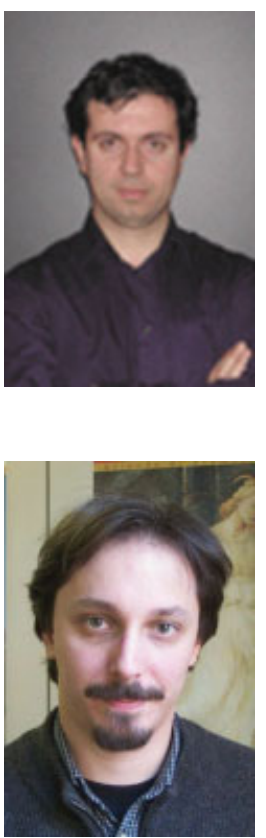

Dr. Tommaso Pecorella was born in Firenze, Italy. He received the Dr.Ing. degree in electronics engineering from the University of Firenze, Firenze, Italy, in 1996, and the $\mathrm{PhD}$ degree in telecommunications engineering in 1999. In 2000 he joined the CNIT - Italian University Consortium for Telecommunications as a researcher. In 2006 he joined the Dipartimento di Elettronica e Telecomunicazioni of University of Firenze as an assistant professor. He has been involved in a number of national end European projects including various COST actions and EU NoEs and projects. In particular he is leader of the WP 2410 - Access, Network and Transport Layer Trials in the SatNExII EU NoE. His research interests involve computer communications, mobile communication networks, QoS-enabled access schemes, satellite communication networks and queuing theory.

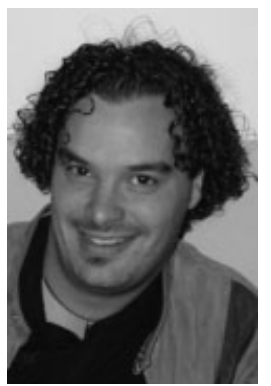

Paolo Barsocchi received his M.S. degree in telecommunication engineering from the University of Pisa (Italy) in 2003.During his Ph.D. he has been working with WnLab research group at the ISTI, an Institute of the Italian National Research Council (CNR), where he is currently a post-doctoral fellow. His research interests are sensor network, wireless channel modelling and multimedia communications and services in terrestrial wireless networks. 


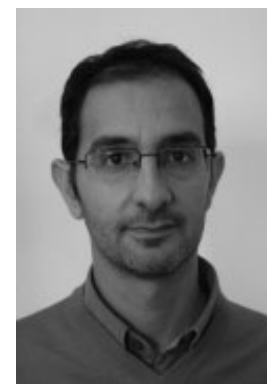

Stefano chessa received his MSc and PhD - degrees in Computer Science from the University of Pisa, Italy, in 1994 and 1999, respectively.

Currently he is assistant professor at the Department of Computer Science of the University of Pisa and also Research Associate at the ISTI/CNR Institute. His main research interests are in the areas of ad hoc and wireless sensor networks and video streams authentication.

He is involved in the EU FP6 Networks of Excellence SatNex and InterMedia, the FP6 Strep project SMEPP, and in the FP6 Integrated Project PERSONA. He has coauthored more than 50 papers published on international journals and conference proceedings.

Dr. Erina Ferro received her Laurea degree with distinction in Computer Science from the University of Pisa, Italy, in 1975. Since 1976 Dr. Ferro was with CNR (National Research Council), and she is currently employed as a Director of Research at the Institute CNR-ISTI. The National Research Council (CNR) is the governmental Italian research organization, whose headquarter is in Rome. The CNR Scientific Network is constituted by about 107 Institutes, aggregated in 11 thematic Departments. ISTI institute belongs to the ICT (Information and Communication Technologies) Department of CNR.

In 1980 Dr. Ferro started working on digital satellite communications. She participated to several international and national projects where some systems co-designed by her were implemented and used on the Eutelsat, Olympus, and Italsat satellites. Working in the TDMA satellite access schemes, field she obtained two patents, in 1989 and 1996, respectively, together with other colleagues of her group.

Dr. Ferro is head of the Wireless Networks Laboratory (WNLAB) at ISTI, and since 2006 she is responsible of the Devices and Technologies for Telematic Networks scientific sector of the ICT Department of CNR (www.ict.cnr.it). Her main research activities cover sensor networks, wireless networks, ad-hoc networks, satellite networks, and the interconnection aspects. She is associated editor of the International Journals on Communication Systems (IJCS). She is reviewer for several international journals and congresses. She authored about hundred international journal and congress papers.

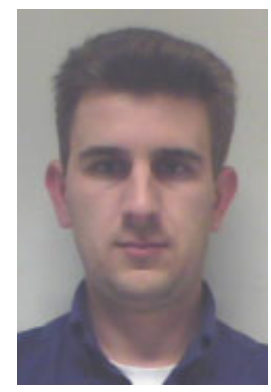

Gabriele Oligeri is a researcher in the Wireless Network Laboratory at ISTI, an Institute of the Italian National Research Council (CNR) since 2005. His research focuses on security on wireless networks, and wireless channel modelling. $\mathrm{He}$ received the M. Eng. degree in Computer Engineering from the University of Pisa, Italy, in 2005 . He is currently a PhD student in Information Engineering at the University of Pisa.

Francesco Potortí is a senior researcher at the ISTI institute of CNR in Pisa, Italy. His interests include wireless communications, network simulation and free software. Home page at $<$ http://fly.isti.cnr.it $>$. 


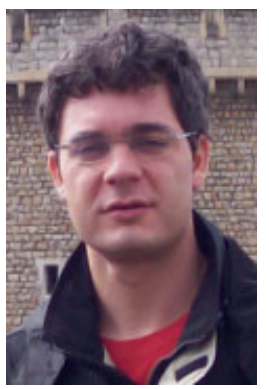

Secchi Raffaello received his Laurea degree in Telecommunication Engineering on July 2002 from the University of Pisa (Italy). From January 2003 to December 2005 he was a $\mathrm{PhD}$ student at the University of Pisa and received his $\mathrm{PhD}$ from the on May 2006, by defending a thesis on Traffic Modelling and Control in High Speed Networks. Since 2006 he was a Postdoctoral Fellow with the Information Science and Technology Institute (ISTI) of National Research Council in Pisa, and is currently a Research Fellow at the University of Aberdeen.

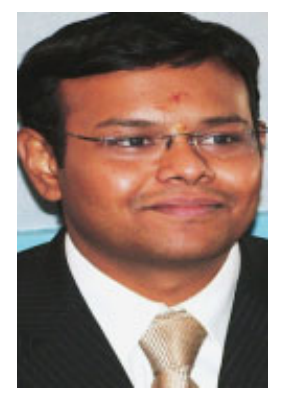

Arjuna Sathiaseelan (PhD 2005, BE 2000) is a Research Fellow at the University of Aberdeen. His current research interests are in the integration of satellite broadband access in Next Generation Networks. He leads work on Network Simulation, design of multimedia transport protocols, TCP protocol optimization, and performance analysis. He is the author of several working group Internet Drafts in the IETF DCCP working group.

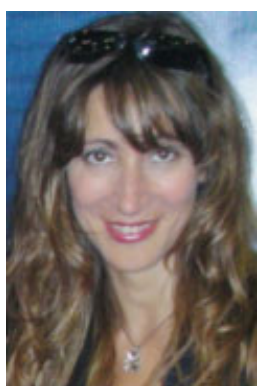

María Angeles Vázquez Castro received the Telecommunication Engineer degree (1994) and PhD (cum laude, 1998) both from the Polytechnic University of Vigo (Spain). She is currently an Associate professor at the Universitat AutEnoma de Barcelona (Spain). She has been a Research Fellow at the European Space Agency (2002-2004) and a visiting researcher at the University of Southern California (2000). She leads the research group on Wireless Communications that belongs to the European Network of Excellence on Satellite Communications. She has lead and participated in national and international research projects and has co-authorised book chapters and around 80 papers, holds one patent and contributes to the standardization bodies ITU, DVB and ETSI. Her current research interests are cross-layer and cooperative design of wireless terrestrial and satellite systems.

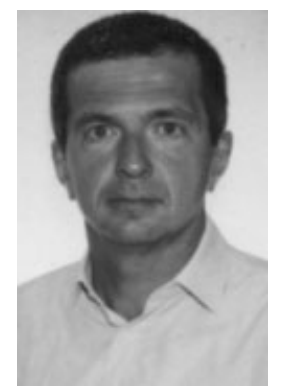

Carlo Caini received the Dr. Ing. Degree (summa cum laude) in Electronic Engineering from the University of Bologna, Italy, in 1986. Since 1990, he has been with the Department of Electronics Computer Science and Systems of the same University, where he is currently an Associate Professor. His main scientific interests are in the field of terrestrial and satellite cellular mobile radio systems, with a special emphasis on spectrum efficiency, multiple access techniques and spread spectrum systems. The recent integration of Internet and wireless communications has led him to devote his recent research activity to the development of network protocols and architecture for satellite and wireless applications. He participates to several international research projects and he is author of many international publications on these topics. He is member of IEEE Communications Society. 


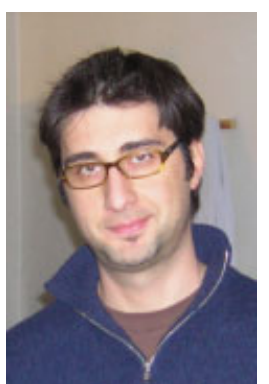

Rosario Firrincieli is a senior researcher at the Advanced Research Center on Electronics Systems for Information and Communication Technologies (ARCES), University of Bologna, Italy. He received his Masters and $\mathrm{PhD}$ degrees in Telecommunications Engineering from the University of Bologna in 2001 and 2006 respectively. During 2005, 2006 and 2007 he spent 10 months as Visiting Researcher at Department of Computer Science, the Henry Samueli School of Engineering and Applied Sciences, UCLA, US. His present interests involve the study and the evaluation of enhanced transport protocols, Performance Enhancing Proxies solutions, and Delay/Disruption Tolerant Networks architectures over wireless network. Moreover, he is interested in congestion control algorithms for unicast and multicast protocols, traffic shaping, retransmission techniques (ARQ, Data Carousel), and packet coding at the transport layer. He is co-author of 30 scientific publications on International Journals and Conferences. 